\title{
The Universal Askey-Wilson Algebra and the Equitable Presentation of $U_{q}\left(\mathfrak{s l}_{2}\right)$
}

\author{
Paul TERWILLIGER
}

Department of Mathematics, University of Wisconsin, Madison, WI 53706-1388, USA

E-mail: terwilli@math.wisc.edu

Received July 19, 2011, in final form October 10, 2011; Published online October 25, 2011

http://dx.doi.org/10.3842/SIGMA.2011.099

\begin{abstract}
Let $\mathbb{F}$ denote a field, and fix a nonzero $q \in \mathbb{F}$ such that $q^{4} \neq 1$. The universal Askey-Wilson algebra is the associative $\mathbb{F}$-algebra $\Delta=\Delta_{q}$ defined by generators and relations in the following way. The generators are $A, B, C$. The relations assert that each of

$$
A+\frac{q B C-q^{-1} C B}{q^{2}-q^{-2}}, \quad B+\frac{q C A-q^{-1} A C}{q^{2}-q^{-2}}, \quad C+\frac{q A B-q^{-1} B A}{q^{2}-q^{-2}}
$$

is central in $\Delta$. In this paper we discuss a connection between $\Delta$ and the $\mathbb{F}$-algebra $U=$ $U_{q}\left(\mathfrak{s l}_{2}\right)$. To summarize the connection, let $a, b, c$ denote mutually commuting indeterminates and let $\mathbb{F}\left[a^{ \pm 1}, b^{ \pm 1}, c^{ \pm 1}\right]$ denote the $\mathbb{F}$-algebra of Laurent polynomials in $a, b, c$ that have all coefficients in $\mathbb{F}$. We display an injection of $\mathbb{F}$-algebras $\Delta \rightarrow U \otimes_{\mathbb{F}} \mathbb{F}\left[a^{ \pm 1}, b^{ \pm 1}, c^{ \pm 1}\right]$. For this injection we give the image of $A, B, C$ and the above three central elements, in terms of the equitable generators for $U$. The algebra $\Delta$ has another central element of interest, called the Casimir element $\Omega$. One significance of $\Omega$ is the following. It is known that the center of $\Delta$ is generated by $\Omega$ and the above three central elements, provided that $q$ is not a root of unity. For the above injection we give the image of $\Omega$ in terms of the equitable generators for $U$. We also use the injection to show that $\Delta$ contains no zero divisors.
\end{abstract}

Key words: Askey-Wilson relations; Leonard pair; Casimir element

2010 Mathematics Subject Classification: 33D80; 33D45

\section{Introduction}

The Askey-Wilson polynomials were introduced in [4] and soon became renown for their algebraic, analytic, and combinatorial properties [7, 11]. In his study [20] of the "hidden symmetry" of these polynomials, A. Zhedanov introduced the Askey-Wilson algebra AW(3). This algebra is defined by generators and relations. The relations involve a nonzero parameter $q$ and 5 additional parameters. The algebra is infinite dimensional and noncommutative. Zhedanov's original presentation involves three generators; however one generator is a $q$-commutator of the other two and is sometimes eliminated. The remaining two generators satisfy a pair of relations often called the Askey-Wilson relations [18]. These relations describe the Askey-Wilson polynomials in the following way. Let $\left\{p_{n}\right\}_{n=0}^{\infty}$ denote a sequence of Askey-Wilson polynomials in a variable $\lambda$. These polynomials are eigenvectors for a certain $q$-difference operator, known as the Askey-Wilson operator and denoted for the moment by $A$. Let $B$ denote the linear operator that sends $p(\lambda) \mapsto \lambda p(\lambda)$ for all polynomials $p(\lambda)$. The operator $B$ acts on the basis $\left\{p_{n}\right\}_{n=0}^{\infty}$ in an irreducible tridiagonal fashion, reflecting the fact that $\left\{p_{n}\right\}_{n=0}^{\infty}$ satisfy a three-term recurrence. In [20] Zhedanov showed that $A, B$ satisfy a pair of Askey-Wilson relations.

Although the Askey-Wilson relations are slightly complicated, over time their significance became clear as they found applications to integrable systems [5, 13, 19], quantum groups [6], linear algebra [15, 18], quantum mechanics [14], and the double affine Hecke algebra [12, 8]. We now describe the two applications most relevant to the present paper. 
Our first application concerns a linear algebraic object called a Leonard pair. This is a pair of diagonalizable linear transformations on a finite-dimensional vector space, each of which acts in an irreducible tridiagonal fashion on an eigenbasis for the other one [15, Definition 1.1]. In [18] Vidunas and the present author showed that a Leonard pair satisfies a pair of Askey-Wilson relations. This example is closely related to the one involving the Askey-Wilson polynomials. By [16, Section 5] the Leonard pairs correspond to a family of orthogonal polynomials consisting of the $q$-Racah polynomials and their relatives. The polynomials in this family are special or limiting cases of the Askey-Wilson polynomials [11].

Our second application is about quantum groups. Consider the algebra $U_{q}\left(\mathfrak{s l}_{2}\right)$ with the usual Chevalley generators $e, f, k^{ \pm 1}[10]$. Let $A$ denote an arbitrary linear combination of $e k^{-1}, f, k^{-1}$ and let $B$ denote an arbitrary linear combination of $e, f k, k$. Then according to Granovskiu and Zhedanov [6] the elements $A, B$ satisfy a pair of Askey-Wilson relations. In [19] Wiegmann and Zabrodin extended this result by displaying an element $C$ in $U_{q}\left(\mathfrak{s l}_{2}\right)$ such that

$$
\begin{aligned}
& q A B-q^{-1} B A=g_{C} C+h_{C}, \\
& q B C-q^{-1} C B=g_{A} A+h_{A}, \\
& q C A-q^{-1} A C=g_{B} B+h_{B},
\end{aligned}
$$

where $g_{A}, g_{B}, g_{C}$ and $h_{A}, h_{B}, h_{C}$ are scalars in the underlying field. The equations (1.1)-(1.3) are often called the $\mathbb{Z}_{3}$-symmetric Askey-Wilson relations [8]. Upon eliminating $C$ in (1.2), (1.3) using (1.1) we obtain the Askey-Wilson relations in the variables $A, B$. Upon substituting $C^{\prime}=$ $g_{C} C+h_{C}$ in (1.1)-(1.3) we recover the original presentation for $\mathrm{AW}(3)$ in the variables $A, B, C^{\prime}$.

We now recall the universal Askey-Wilson algebra $\Delta[17]$. To motivate this algebra consider the relations (1.1)-(1.3). They are attractive but one might object that there are too many parameters. To accomodate this objection we will eliminate all the parameters besides $q$. We will do this without significantly reducing the generality of the algebra (although we allow a minor technical assumption). We first eliminate $g_{A}, g_{B}, g_{C}$ with the following change of variables. Assume that each of $g_{A} g_{B}, g_{B} g_{C}, g_{C} g_{A}$ is a nonzero square in the underlying field, and that $q^{4} \neq 1$. Now in (1.1)-(1.3) replace $A, B, C$ by

$$
\frac{A f_{A}}{q^{-2}-q^{2}}, \quad \frac{B f_{B}}{q^{-2}-q^{2}}, \quad \frac{C f_{C}}{q^{-2}-q^{2}}
$$

respectively, where

$$
f_{A}^{2}=g_{B} g_{C}, \quad f_{B}^{2}=g_{C} g_{A}, \quad f_{C}^{2}=g_{A} g_{B}, \quad f_{A} f_{B} f_{C}=g_{A} g_{B} g_{C} .
$$

The resulting equations assert that each of

$$
A+\frac{q B C-q^{-1} C B}{q^{2}-q^{-2}}, \quad B+\frac{q C A-q^{-1} A C}{q^{2}-q^{-2}}, \quad C+\frac{q A B-q^{-1} B A}{q^{2}-q^{-2}}
$$

is a scalar in the underlying field. We have eliminated $g_{A}, g_{B}, g_{C}$ and are now down to the three scalar parameters (1.4). To eliminate these we reinterpret them as central elements in the algebra generated by $A, B, C$. The resulting algebra is denoted $\Delta$ and called the universal Askey-Wilson algebra [17]. The formal definition of $\Delta$ is given in Definition 2.1 below.

In [17] we investigated $\Delta$ from a ring theoretic point of view. Our results include the following. We displayed a faithful action of the modular group $\mathrm{PSL}_{2}(\mathbb{Z})$ on $\Delta$ as a group of automorphisms [17, Theorems 3.1, 3.13]. We found several linear bases for $\Delta[17$, Theorems 4.1, 7.5]. We described the center $Z(\Delta)$ under the assumption that $q$ is not a root of unity. For such $q$ we found that $Z(\Delta)$ is generated by the three central elements (1.4) together with an element $\Omega$ called the Casimir element [17, Corollary 8.3]. 
We now discuss the equitable presentation for $U_{q}\left(\mathfrak{s l}_{2}\right)$ [9]. This presentation involves generators $x, y^{ \pm 1}, z$ and relations $y y^{-1}=y^{-1} y=1$,

$$
\frac{q x y-q^{-1} y x}{q-q^{-1}}=1, \quad \frac{q y z-q^{-1} z y}{q-q^{-1}}=1, \quad \frac{q z x-q^{-1} x z}{q-q^{-1}}=1 .
$$

In [1] H. AlNajjar investigated Leonard pairs using the equitable presentation of $U_{q}\left(\mathfrak{s l}_{2}\right)$. His approach is summarized as follows. Let $V$ denote a finite-dimensional irreducible $U_{q}\left(\mathfrak{s l}_{2}\right)$-module. Let $A$ denote an arbitrary linear combination of $1, x, y, x y$ and let $B$ denote an arbitrary linear combination of $1, y, z, y z$. Consider the coefficients. Alnajjar found necessary and sufficient conditions on the coefficients for $A, B$ to act on $V$ as a Leonard pair [1, Theorem 6.2]. In [2] Alnajjar described the class of Leonard pairs that result from his construction. He showed that this class corresponds to a family of orthogonal polynomials consisting of the $q$-Racah, $q$-Hahn, dual $q$-Hahn, $q$-Krawtchouk, dual $q$-Krawtchouk, affine $q$-Krawtchouk, and quantum $q$-Krawtchouk polynomials. For the Leonard pairs $A, B$ in the above class consider the corresponding AskeyWilson relations. We use the $\mathbb{Z}_{3}$-symmetric version in view of the $\mathbb{Z}_{3}$-symmetric nature of the equitable presentation. In the style of Wiegmann and Zabrodin let $C$ denote an arbitrary linear combination of $1, z, x, z x$ and consider when $A, B, C$ satisfy some $\mathbb{Z}_{3}$-symmetric Askey-Wilson relations. Extending the work of Alnajjar one finds that the "most general" solution is described as follows.

Proposition 1.1. Let $\mathbb{F}$ denote a field, and fix a nonzero $q \in \mathbb{F}$ such that $q^{4} \neq 1$. Consider the $\mathbb{F}$-algebra $U_{q}\left(\mathfrak{s l}_{2}\right)$ with equitable generators $x, y^{ \pm 1}$, z. Let $a, b, c$ denote nonzero scalars in $\mathbb{F}$ and define

$$
\begin{aligned}
& A=x a+y a^{-1}+\frac{x y-y x}{q-q^{-1}} b c^{-1}, \\
& B=y b+z b^{-1}+\frac{y z-z y}{q-q^{-1}} c a^{-1}, \\
& C=z c+x c^{-1}+\frac{z x-x z}{q-q^{-1}} a b^{-1} .
\end{aligned}
$$

Then

$$
\begin{aligned}
& A+\frac{q B C-q^{-1} C B}{q^{2}-q^{-2}}=\frac{\Lambda\left(a+a^{-1}\right)+\left(b+b^{-1}\right)\left(c+c^{-1}\right)}{q+q^{-1}}, \\
& B+\frac{q C A-q^{-1} A C}{q^{2}-q^{-2}}=\frac{\Lambda\left(b+b^{-1}\right)+\left(c+c^{-1}\right)\left(a+a^{-1}\right)}{q+q^{-1}}, \\
& C+\frac{q A B-q^{-1} B A}{q^{2}-q^{-2}}=\frac{\Lambda\left(c+c^{-1}\right)+\left(a+a^{-1}\right)\left(b+b^{-1}\right)}{q+q^{-1}} .
\end{aligned}
$$

Here $\Lambda$ denotes the normalized Casimir element of $U_{q}\left(\mathfrak{s l}_{2}\right)$ from Lemma 2.15 below.

Let $q, a, b, c$ be from Proposition 1.1. By that proposition and since $\Lambda$ is central in $U_{q}\left(\mathfrak{s l}_{2}\right)$, there exists an algebra homomorphism $\Delta \rightarrow U_{q}\left(\mathfrak{s l}_{2}\right)$ that acts on the $\Delta$-generators $A, B, C$ in the following way. It sends

$$
\begin{aligned}
& A \mapsto x a+y a^{-1}+\frac{x y-y x}{q-q^{-1}} b c^{-1}, \\
& B \mapsto y b+z b^{-1}+\frac{y z-z y}{q-q^{-1}} c a^{-1}, \\
& C \mapsto z c+x c^{-1}+\frac{z x-x z}{q-q^{-1}} a b^{-1} .
\end{aligned}
$$


It turns out that this homomorphism is not injective. In order to shrink the kernel we reinterpret $a, b, c$ as mutually commuting indeterminates, and view the above construction as giving an algebra homomorphism $\Delta \mapsto U_{q}\left(\mathfrak{s l}_{2}\right) \otimes_{\mathbb{F}} \mathbb{F}\left[a^{ \pm 1}, b^{ \pm 1}, c^{ \pm 1}\right]$. A main result of the present paper is that this homomorphism is injective. In another main result we compute the image of $\Omega$ under the injection. We also use the injection to show that $\Delta$ contains no zero divisors.

The paper is organized as follows. In Section 2 we recall some basic facts and then state our main results, which are Theorems 2.16-2.18 and Corollary 2.19. In Section 3 we establish some identities involving the equitable generators of $U_{q}\left(\mathfrak{s l}_{2}\right)$, which will be used repeatedly. In Section 4 we prove Theorem 2.16. In Section 5 we prove Theorem 2.17. In Sections 6-8 we establish some slightly technical facts about $U_{q}\left(\mathfrak{s l}_{2}\right)$, which will be used in Section 9 to prove Theorem 2.18. In Section 10 we discuss some issues concerning the $\mathrm{PSL}_{2}(\mathbb{Z})$ action on $\Delta$ that we mentioned earlier.

Our proofs for Theorems 2.16-2.18 are essentially self contained and do not assume Proposition 1.1. We remark that Proposition 1.1 follows from Theorem 2.16.

For the rest of this paper $a, b, c$ denote mutually commuting indeterminates.

\section{Statement of results}

Our conventions for the paper are as follows. An algebra is meant to be associative and have a 1. A subalgebra has the same 1 as the parent algebra. We fix a field $\mathbb{F}$. All unadorned tensor products are meant to be over $\mathbb{F}$. We fix $q \in \mathbb{F}$ such that $q^{4} \neq 1$. Recall the natural numbers $\mathbb{N}=\{0,1,2, \ldots\}$ and integers $\mathbb{Z}=\{0, \pm 1, \pm 2, \ldots\}$.

Definition 2.1 ([17, Definition 1.2]). Define an $\mathbb{F}$-algebra $\Delta=\Delta_{q}$ by generators and relations in the following way. The generators are $A, B, C$. The relations assert that each of

$$
A+\frac{q B C-q^{-1} C B}{q^{2}-q^{-2}}, \quad B+\frac{q C A-q^{-1} A C}{q^{2}-q^{-2}}, \quad C+\frac{q A B-q^{-1} B A}{q^{2}-q^{-2}}
$$

is central in $\Delta$. The algebra $\Delta$ is called the universal Askey-Wilson algebra.

Definition 2.2 ([17, Definition 1.3]). For the three central elements in (2.1), multiply each by $q+q^{-1}$ to get $\alpha, \beta, \gamma$. Thus

$$
\begin{aligned}
& A+\frac{q B C-q^{-1} C B}{q^{2}-q^{-2}}=\frac{\alpha}{q+q^{-1}}, \\
& B+\frac{q C A-q^{-1} A C}{q^{2}-q^{-2}}=\frac{\beta}{q+q^{-1}}, \\
& C+\frac{q A B-q^{-1} B A}{q^{2}-q^{-2}}=\frac{\gamma}{q+q^{-1}} .
\end{aligned}
$$

Note that each of $\alpha, \beta, \gamma$ is central in $\Delta$.

We mention a few facts about $\Delta$. Recall that the modular group $\mathrm{PSL}_{2}(\mathbb{Z})$ has a presentation by generators $\rho, \sigma$ and relations $\rho^{3}=1, \sigma^{2}=1$. See for example [3]. By [17, Theorem 3.1], the group $\operatorname{PSL}_{2}(\mathbb{Z})$ acts on $\Delta$ as a group of automorphisms such that $\rho$ sends $(A, B, C) \mapsto(B, C, A)$ and $\sigma$ sends $(A, B, \gamma) \mapsto(B, A, \gamma)$. By [17, Theorem 3.13] this action is faithful.

By [17, Theorem 4.1] the following is a basis for the $\mathbb{F}$-vector space $\Delta$ :

$$
A^{i} B^{j} C^{k} \alpha^{r} \beta^{s} \gamma^{t}, \quad i, j, k, r, s, t \in \mathbb{N} .
$$

There is a related basis [17, Theorem 7.5] that we will use in Section 9 below. This related basis involves a central element $\Omega$ known as the Casimir element [17, Lemma 6.1]. This element is defined as follows. 
Definition 2.3 ([17, Lemma 6.1]). Define $\Omega \in \Delta$ by

$$
\Omega=q A B C+q^{2} A^{2}+q^{-2} B^{2}+q^{2} C^{2}-q A \alpha-q^{-1} B \beta-q C \gamma
$$

We call $\Omega$ the Casimir element of $\Delta$.

Lemma 2.4 ([17, Theorem 6.2, Corollary 8.3]). The Casimir element $\Omega$ is contained in the center $Z(\Delta)$. Moreover $\left\{\Omega^{i} \alpha^{r} \beta^{s} \gamma^{t} \mid i, r, s, t \in \mathbb{N}\right\}$ is a basis for the $\mathbb{F}$-vector space $Z(\Delta)$, provided that $q$ is not a root of unity.

Lemma 2.5 ([17, Theorem 6.4]). The Casimir element $\Omega$ is fixed by everything in $\mathrm{PSL}_{2}(\mathbb{Z})$.

We will be discussing how $\Delta$ is related to the quantum universal enveloping algebra $U_{q}\left(\mathfrak{s l}_{2}\right)$. For this algebra there are two presentations of interest to us; the Chevalley presentation [10, Section 1.1] and the equitable presentation [9]. We now recall the Chevalley presentation.

Definition 2.6 ([10, Section 1.1]). The $\mathbb{F}$-algebra $U=U_{q}\left(\mathfrak{s l}_{2}\right)$ is defined by generators $e, f$, $k^{ \pm 1}$ and relations

$$
k k^{-1}=k^{-1} k=1, \quad k e=q^{2} e k, \quad k f=q^{-2} f k, \quad \text { ef }-f e=\frac{k-k^{-1}}{q-q^{-1}} .
$$

We call $e, f, k^{ \pm 1}$ the Chevalley generators for $U$.

We now briefly discuss some finite-dimensional $U$-modules. Strictly speaking we will not use this information; it is included in order to clarify the nature of the Casimir element for $U$ described below.

Recall the notation

$$
[n]_{q}=\frac{q^{n}-q^{-n}}{q-q^{-1}}, \quad n \in \mathbb{N}
$$

Lemma 2.7 ([10, Section 2]). For all integers $n \geq 0$ and $\varepsilon \in\{1,-1\}$ there exists a $U$-module $L(n, \varepsilon)$ with the following properties. $L(n, \varepsilon)$ has a basis $\left\{v_{i}\right\}_{i=0}^{n}$ such that

$$
\begin{aligned}
& k v_{i}=\varepsilon q^{n-2 i} v_{i}, \quad 0 \leq i \leq n, \\
& f v_{i}=[i+1]_{q} v_{i+1}, \quad 0 \leq i \leq n-1, \quad f v_{n}=0, \\
& e v_{i}=\varepsilon[n-i+1]_{q} v_{i-1}, \quad 1 \leq i \leq n, \quad e v_{0}=0 .
\end{aligned}
$$

The $U$-module $L(n, \varepsilon)$ is irreducible provided that $q$ is not a root of unity.

In Definition 2.3 we gave the Casimir element for $\Delta$. We now recall the Casimir element for $U$.

Definition 2.8 ([10, Section 2.7]). Define $\Phi \in U$ as follows:

$$
\Phi=e f+\frac{q^{-1} k+q k^{-1}}{\left(q-q^{-1}\right)^{2}} .
$$

We call $\Phi$ the Casimir element of $U$.

Lemma 2.9 ([10, Lemma 2.7, Proposition 2.18]). The element $\Phi$ is contained in the center $Z(U)$. Moreover $\left\{\Phi^{i}\right\}_{i \in \mathbb{N}}$ is a basis for the $\mathbb{F}$-vector space $Z(U)$, provided that $q$ is not a root of unity. 
Lemma 2.10. [10, Lemma 2.7]. On the $U$-module $L(n, \varepsilon)$,

$$
\Phi=\varepsilon \frac{q^{n+1}+q^{-n-1}}{\left(q-q^{-1}\right)^{2}} I .
$$

Here I denotes the identity map.

For notational convenience we now adjust the normalization for $\Phi$.

Definition 2.11. Define

$$
\Lambda=\left(q-q^{-1}\right)^{2} \Phi=\left(q-q^{-1}\right)^{2} e f+q^{-1} k+q k^{-1} .
$$

Note that on $L(n, \varepsilon)$,

$$
\Lambda=\varepsilon\left(q^{n+1}+q^{-n-1}\right) I .
$$

We call $\Lambda$ the normalized Casimir element for $U$.

We now recall the equitable presentation for $U$ [9].

Proposition 2.12 ([9, Theorem 2.1]). The algebra $U$ is isomorphic to the $\mathbb{F}$-algebra defined by generators $x, y^{ \pm 1}, z$ and relations

$$
\begin{aligned}
& y y^{-1}=y^{-1} y=1, \\
& \frac{q x y-q^{-1} y x}{q-q^{-1}}=1, \\
& \frac{q y z-q^{-1} z y}{q-q^{-1}}=1, \\
& \frac{q z x-q^{-1} x z}{q-q^{-1}}=1 .
\end{aligned}
$$

An isomorphism with the presentation in Definition 2.6 is given by

$$
y^{ \pm 1} \mapsto k^{ \pm 1}, \quad z \mapsto k^{-1}+f\left(q-q^{-1}\right), \quad x \mapsto k^{-1}-e k^{-1} q^{-1}\left(q-q^{-1}\right)
$$

The inverse of this isomorphism is given by

$$
k^{ \pm 1} \mapsto y^{ \pm 1}, \quad f \mapsto\left(z-y^{-1}\right)\left(q-q^{-1}\right)^{-1}, \quad e \mapsto(1-x y) q\left(q-q^{-1}\right)^{-1} .
$$

Definition 2.13 ([9, Definition 2.2]). By the equitable presentation of $U$ we mean the presentation given in Proposition 2.12. We call $x, y^{ \pm 1}, z$ the equitable generators for $U$.

Note 2.14. In what follows we identify the copy of $U$ given in Definition 2.6 with the copy given in Proposition 2.12, via the isomorphism given in Proposition 2.12.

In the equitable presentation of $U$ the normalized Casimir element $\Lambda$ looks as follows.

Lemma 2.15. The normalized Casimir element $\Lambda$ is equal to each of the following:

$$
\begin{array}{ll}
q x+q^{-1} y+q z-q x y z, & q^{-1} x+q y+q^{-1} z-q^{-1} z y x, \\
q y+q^{-1} z+q x-q y z x, & q^{-1} y+q z+q^{-1} x-q^{-1} x z y, \\
q z+q^{-1} x+q y-q z x y, & q^{-1} z+q x+q^{-1} y-q^{-1} y x z .
\end{array}
$$


Proof. For the data (2.10)-(2.12) let $\Lambda_{y}^{-}, \Lambda_{z}^{-}, \Lambda_{x}^{-}$denote the expressions in the first column and let $\Lambda_{y}^{+}, \Lambda_{z}^{+}, \Lambda_{x}^{+}$denote the expressions in the second column. Consider the expression for $\Lambda$ given in (2.5). Writing this expression in terms of $x, y, z$ using the isomorphism in Proposition 2.12 and Note 2.14 , we obtain $\Lambda=\Lambda_{y}^{-}$. The element $\Lambda_{y}^{-}-\Lambda_{z}^{+}$is equal to $\left(q-q^{-1}\right) x$ times

$$
1-\frac{q y z-q^{-1} z y}{q-q^{-1}}
$$

The expression (2.13) is zero by (2.8) so $\Lambda_{y}^{-}=\Lambda_{z}^{+}$. Similarly one finds $\Lambda_{z}^{-}=\Lambda_{x}^{+}$and $\Lambda_{x}^{-}=\Lambda_{y}^{+}$. The element $\Lambda_{y}^{-}-\Lambda_{x}^{+}$is equal to

$$
1-\frac{q x y-q^{-1} y x}{q-q^{-1}}
$$

times $\left(q-q^{-1}\right) z$. The expression (2.14) is zero by (2.7) so $\Lambda_{y}^{-}=\Lambda_{x}^{+}$. Similarly one finds $\Lambda_{z}^{-}=\Lambda_{y}^{+}$and $\Lambda_{x}^{-}=\Lambda_{z}^{+}$. By these comments $\Lambda$ is equal to each of $\Lambda_{x}^{ \pm}, \Lambda_{y}^{ \pm}, \Lambda_{z}^{ \pm}$.

Recall that $a, b, c$ are mutually commuting indeterminates. Let $\mathbb{F}\left[a^{ \pm 1}, b^{ \pm 1}, c^{ \pm 1}\right]$ denote the $\mathbb{F}$-algebra of Laurent polynomials in $a, b, c$ that have all coefficients in $\mathbb{F}$.

We now state our main results.

Theorem 2.16. There exists a unique $\mathbb{F}$-algebra homomorphism $\natural: \Delta \rightarrow U \otimes \mathbb{F}\left[a^{ \pm 1}, b^{ \pm 1}, c^{ \pm 1}\right]$ that sends

$$
\begin{aligned}
& A \mapsto x \otimes a+y \otimes a^{-1}+\frac{x y-y x}{q-q^{-1}} \otimes b c^{-1}, \\
& B \mapsto y \otimes b+z \otimes b^{-1}+\frac{y z-z y}{q-q^{-1}} \otimes c a^{-1}, \\
& C \mapsto z \otimes c+x \otimes c^{-1}+\frac{z x-x z}{q-q^{-1}} \otimes a b^{-1},
\end{aligned}
$$

where $x, y, z$ denote the equitable generators for $U$. The homomorphism $\downarrow$ sends

$$
\begin{aligned}
& \alpha \mapsto \Lambda \otimes\left(a+a^{-1}\right)+1 \otimes\left(b+b^{-1}\right)\left(c+c^{-1}\right), \\
& \beta \mapsto \Lambda \otimes\left(b+b^{-1}\right)+1 \otimes\left(c+c^{-1}\right)\left(a+a^{-1}\right), \\
& \gamma \mapsto \Lambda \otimes\left(c+c^{-1}\right)+1 \otimes\left(a+a^{-1}\right)\left(b+b^{-1}\right),
\end{aligned}
$$

where $\Lambda$ denotes the normalized Casimir element of $U$.

Theorem 2.17. Under the homomorphism $\boxminus$ from Theorem 2.16, the image of $\Omega$ is

$$
\begin{gathered}
1 \otimes\left(q+q^{-1}\right)^{2}-1 \otimes\left(a+a^{-1}\right)^{2}-1 \otimes\left(b+b^{-1}\right)^{2}-1 \otimes\left(c+c^{-1}\right)^{2} \\
-\Lambda \otimes\left(a+a^{-1}\right)\left(b+b^{-1}\right)\left(c+c^{-1}\right)-\Lambda^{2} \otimes 1 .
\end{gathered}
$$

Here $\Lambda$ denotes the normalized Casimir element of $U$.

Theorem 2.18. The homomorphism $\downarrow$ from Theorem 2.16 is injective.

We mention a corollary to Theorem 2.18. For an $\mathbb{F}$-algebra $\mathcal{A}$, an element $u \in \mathcal{A}$ is called a zero divisor whenever $u \neq 0$ and there exists $0 \neq v \in \mathcal{A}$ such that $u v=0$. By [10, Proposition 1.8] the algebra $U$ contains no zero divisors. For an $\mathbb{F}$-algebra $\mathcal{A}$ and indeterminate $\lambda$ consider the $\mathbb{F}$-algebra $\mathcal{A} \otimes \mathbb{F}\left[\lambda, \lambda^{-1}\right]$. One checks that $\mathcal{A}$ contains no zero divisors if and only if $\mathcal{A} \otimes \mathbb{F}\left[\lambda, \lambda^{-1}\right]$ contains no zero divisors. Applying this comment three times we see that the algebra $U \otimes$ $\mathbb{F}\left[a^{ \pm 1}, b^{ \pm 1}, c^{ \pm 1}\right]$ contains no zero divisors. By this and Theorem 2.18 we obtain the following result.

Corollary 2.19. The $\mathbb{F}$-algebra $\Delta$ contains no zero divisors. 


\section{The elements $\nu_{x}, \nu_{y}, \nu_{z}$}

In this section we record a number of identities involving the equitable generators for $U$. These identities will be used in our proof of Theorems 2.16-2.18.

The relations (2.7)-(2.9) can be reformulated as follows:

$$
q(1-y z)=q^{-1}(1-z y), \quad q(1-z x)=q^{-1}(1-x z), \quad q(1-x y)=q^{-1}(1-y x) .
$$

Definition 3.1. Let $\nu_{x}, \nu_{y}, \nu_{z}$ denote the following elements in $U$ :

$$
\begin{aligned}
& \nu_{x}=q(1-y z)=q^{-1}(1-z y), \\
& \nu_{y}=q(1-z x)=q^{-1}(1-x z), \\
& \nu_{z}=q(1-x y)=q^{-1}(1-y x) .
\end{aligned}
$$

Note 3.2. We have

$$
e=\frac{\nu_{z}}{q-q^{-1}}, \quad f=-\frac{q^{-1} y^{-1} \nu_{x}}{q-q^{-1}}, \quad \nu_{z}=\left(q-q^{-1}\right) e, \quad \nu_{x}=-q\left(q-q^{-1}\right) k f .
$$

Lemma 3.3. The following relations hold in $U$ :

$$
\begin{aligned}
& x y=1-q^{-1} \nu_{z}, \quad y x=1-q \nu_{z}, \\
& y z=1-q^{-1} \nu_{x}, \quad z y=1-q \nu_{x}, \\
& z x=1-q^{-1} \nu_{y}, \quad x z=1-q \nu_{y} .
\end{aligned}
$$

Proof. These equations are reformulations of (3.1)-(3.3).

Lemma 3.4. The following relations hold in $U$ :

$$
\begin{array}{ll}
\frac{x y-y x}{q-q^{-1}}=\nu_{z}, & \frac{q y x-q^{-1} x y}{q-q^{-1}}=1-\left(q+q^{-1}\right) \nu_{z}, \\
\frac{y z-z y}{q-q^{-1}}=\nu_{x}, & \frac{q z y-q^{-1} y z}{q-q^{-1}}=1-\left(q+q^{-1}\right) \nu_{x}, \\
\frac{z x-x z}{q-q^{-1}}=\nu_{y}, & \frac{q x z-q^{-1} z x}{q-q^{-1}}=1-\left(q+q^{-1}\right) \nu_{y} .
\end{array}
$$

Proof. For each equation evaluate the left-hand side using Lemma 3.3.

Lemma 3.5. The following relations hold in $U$ :

$$
\begin{array}{ll}
x \nu_{y}=q^{2} \nu_{y} x, & x \nu_{z}=q^{-2} \nu_{z} x, \\
y \nu_{z}=q^{2} \nu_{z} y, & y \nu_{x}=q^{-2} \nu_{x} y, \\
z \nu_{x}=q^{2} \nu_{x} z, & z \nu_{y}=q^{-2} \nu_{y} z .
\end{array}
$$

Proof. Using $\nu_{y}=q(1-z x)$ we find $q^{-1} x \nu_{y}=x-x z x$. Using $\nu_{y}=q^{-1}(1-x z)$ we find $q \nu_{y} x=x-x z x$. By these comments $x \nu_{y}=q^{2} \nu_{y} x$. The remaining relations are similarly obtained.

Lemma 3.6. The following relations hold in $U$ :

$$
\begin{array}{ll}
\nu_{x} x=\Lambda-q y-q^{-1} z, & x \nu_{x}=\Lambda-q^{-1} y-q z, \\
\nu_{y} y=\Lambda-q z-q^{-1} x, & y \nu_{y}=\Lambda-q^{-1} z-q x, \\
\nu_{z} z=\Lambda-q x-q^{-1} y, & z \nu_{z}=\Lambda-q^{-1} x-q y .
\end{array}
$$


Proof. To verify the equation on the left in (3.13), eliminate $\nu_{x}$ using $\nu_{x}=q(1-y z)$, and eliminate $\Lambda$ using the fact that $\Lambda$ is equal to the expression on the left in (2.11). The remaining equations are similarly verified.

Lemma 3.7. The following relations hold in $U$ :

$$
\frac{x \nu_{x}-\nu_{x} x}{q-q^{-1}}=y-z, \quad \frac{y \nu_{y}-\nu_{y} y}{q-q^{-1}}=z-x, \quad \frac{z \nu_{z}-\nu_{z} z}{q-q^{-1}}=x-y .
$$

Proof. For each equation evaluate the left-hand side using Lemma 3.6.

Lemma 3.8. The normalized Casimir element $\Lambda$ is equal to each of the following:

$$
\begin{array}{ll}
\frac{q x \nu_{x}-q^{-1} \nu_{x} x}{q-q^{-1}}+\left(q+q^{-1}\right) z, & \frac{q \nu_{x} x-q^{-1} x \nu_{x}}{q-q^{-1}}+\left(q+q^{-1}\right) y, \\
\frac{q y \nu_{y}-q^{-1} \nu_{y} y}{q-q^{-1}}+\left(q+q^{-1}\right) x, & \frac{q \nu_{y} y-q^{-1} y \nu_{y}}{q-q^{-1}}+\left(q+q^{-1}\right) z, \\
\frac{q z \nu_{z}-q^{-1} \nu_{z} z}{q-q^{-1}}+\left(q+q^{-1}\right) y, & \frac{q \nu_{z} z-q^{-1} z \nu_{z}}{q-q^{-1}}+\left(q+q^{-1}\right) x .
\end{array}
$$

Proof. Evaluate each of the displayed expressions using Lemma 3.6.

Lemma 3.9. The following relations hold in $U$ :

$$
\begin{array}{ll}
\nu_{x} \nu_{y}=1-q^{-1} \Lambda z+q^{-2} z^{2}, & \nu_{y} \nu_{x}=1-q \Lambda z+q^{2} z^{2}, \\
\nu_{y} \nu_{z}=1-q^{-1} \Lambda x+q^{-2} x^{2}, & \nu_{z} \nu_{y}=1-q \Lambda x+q^{2} x^{2}, \\
\nu_{z} \nu_{x}=1-q^{-1} \Lambda y+q^{-2} y^{2}, & \nu_{x} \nu_{z}=1-q \Lambda y+q^{2} y^{2} .
\end{array}
$$

Proof. To get the equation on the left in (3.19), observe

$$
\begin{aligned}
\nu_{x} \nu_{y} & =q^{-1} \nu_{x}(1-x z)=q^{-1} \nu_{x}-q^{-1} \nu_{x} x z=1-y z-q^{-1}\left(\Lambda-q y-q^{-1} z\right) z \\
& =1-q^{-1} \Lambda z+q^{-2} z^{2} .
\end{aligned}
$$

The remaining equations are similarly verified.

Lemma 3.10. The following relations hold in $U$ :

$$
\begin{aligned}
& \frac{q \nu_{x} \nu_{y}-q^{-1} \nu_{y} \nu_{x}}{q-q^{-1}}=1-z^{2}, \\
& \frac{q \nu_{y} \nu_{z}-q^{-1} \nu_{z} \nu_{y}}{q-q^{-1}}=1-x^{2}, \\
& \frac{q \nu_{z} \nu_{x}-q^{-1} \nu_{x} \nu_{z}}{q-q^{-1}}=1-y^{2} .
\end{aligned}
$$

Proof. For each equation evaluate the left-hand side using Lemma 3.9.

\section{The proof of Theorem 2.16}

In this section we prove Theorem 2.16.

For notational convenience we define some elements in $U \otimes \mathbb{F}\left[a^{ \pm 1}, b^{ \pm 1}, c^{ \pm 1}\right]$ :

$$
\begin{aligned}
& A^{\natural}=x \otimes a+y \otimes a^{-1}+\nu_{z} \otimes b c^{-1}, \\
& B^{\natural}=y \otimes b+z \otimes b^{-1}+\nu_{x} \otimes c a^{-1},
\end{aligned}
$$




$$
C^{\natural}=z \otimes c+x \otimes c^{-1}+\nu_{y} \otimes a b^{-1}
$$

and

$$
\begin{aligned}
& \alpha^{\natural}=\Lambda \otimes\left(a+a^{-1}\right)+1 \otimes\left(b+b^{-1}\right)\left(c+c^{-1}\right), \\
& \beta^{\natural}=\Lambda \otimes\left(b+b^{-1}\right)+1 \otimes\left(c+c^{-1}\right)\left(a+a^{-1}\right), \\
& \gamma^{\natural}=\Lambda \otimes\left(c+c^{-1}\right)+1 \otimes\left(a+a^{-1}\right)\left(b+b^{-1}\right) .
\end{aligned}
$$

Note that each of $\alpha^{\natural}, \beta^{\natural}, \gamma^{\natural}$ is central in $U \otimes \mathbb{F}\left[a^{ \pm 1}, b^{ \pm 1}, c^{ \pm 1}\right]$.

Proof of Theorem 2.16. We first establish the existence of the homomorphism in the theorem statement. To do this it suffices to show that

$$
\begin{aligned}
& A^{\natural}+\frac{q B^{\natural} C^{\natural}-q^{-1} C^{\natural} B^{\natural}}{q^{2}-q^{-2}}=\frac{\alpha^{\natural}}{q+q^{-1}}, \\
& B^{\natural}+\frac{q C^{\natural} A^{\natural}-q^{-1} A^{\natural} C^{\natural}}{q^{2}-q^{-2}}=\frac{\beta^{\natural}}{q+q^{-1}}, \\
& C^{\natural}+\frac{q A^{\natural} B^{\natural}-q^{-1} B^{\natural} A^{\natural}}{q^{2}-q^{-2}}=\frac{\gamma^{\natural}}{q+q^{-1}} .
\end{aligned}
$$

We verify (4.9). Let $P$ denote the left-hand side of (4.9) minus the right-hand side of (4.9). We show that $P=0$. View $P$ as a Laurent polynomial in $a, b, c$ that has coefficients in $U$. We will show that in this polynomial each coefficient is zero. To this end, evaluate $P$ using (4.1)-(4.3), (4.6) and then collect terms. We list below the terms for which the coefficient in $P$ is potentially nonzero:

$$
a b, \quad a b^{-1}, \quad a^{-1} b, \quad a^{-1} b^{-1}, \quad c, \quad c^{-1}, \quad a^{-2} c, \quad b^{2} c^{-1} .
$$

For each of these terms the coefficient in $P$ is listed in the table below, along with a reason why that coefficient is zero.

\begin{tabular}{c|c|c} 
term & coefficient in $P$ & why the coefficient is 0 \\
\hline \hline$a b$ & $\frac{q x y-q^{-1} y x}{q^{2}-q^{-2}}-\frac{1}{q+q^{-1}}$ & equation $(2.7)$ \\
$a b^{-1}$ & $\frac{q x z-q^{-1} z x}{q^{2}-q^{-2}}+\nu_{y}-\frac{1}{q+q^{-1}}$ & equation $(3.9)$ \\
$a^{-1} b$ & $\frac{q\left(y^{2}+\nu_{z} \nu_{x}\right)-q^{-1}\left(y^{2}+\nu_{x} \nu_{z}\right)}{q^{2}-q^{-2}}-\frac{1}{q+q^{-1}}$ & equation $(3.24)$ \\
$a^{-1} b^{-1}$ & $\frac{q y z-q^{-1} z y}{q^{2}-q^{-2}}-\frac{1}{q+q^{-1}}$ & equation $(2.8)$ \\
\hline$c$ & $\frac{q x \nu_{x}-q^{-1} \nu_{x} x}{q^{2}-q^{-2}}+z-\frac{\Lambda}{q+q^{-1}}$ & equation $(3.16)$ \\
$c^{-1}$ & $\frac{q \nu_{z} z-q^{-1} z \nu_{z}}{q^{2}-q^{-2}}+x-\frac{\Lambda}{q+q^{-1}}$ & equation $(3.18)$ \\
\hline$a^{-2} c$ & $\frac{q y \nu_{x}-q^{-1} \nu_{x} y}{q^{2}-q^{-2}}$ & equation $(3.11)$ \\
$b^{2} c^{-1}$ & $\frac{q \nu_{z} y-q^{-1} y \nu_{z}}{q^{2}-q^{-2}}$ & equation $(3.11)$
\end{tabular}

We have shown that for each term in (4.10) the coefficient in $P$ is zero. Therefore $P=0$ and the equation (4.9) holds. The equations (4.7), (4.8) are similarly verified. We have shown that the homomorphism exists. The homomorphism is unique since $A, B, C$ generate $\Delta$. The homomorphism satisfies (2.15)-(2.17) by (4.4)-(4.6) and (4.7)-(4.9). 


\section{The proof of Theorem 2.17}

In this section we prove Theorem 2.17. Recall the Casimir element $\Omega$ from Definition 2.3.

Proof of Theorem 2.17. By Theorem 2.16 the image of $\Omega$ is

$$
q A^{\natural} B^{\natural} C^{\natural}+q^{2}\left(A^{\natural}\right)^{2}+q^{-2}\left(B^{\natural}\right)^{2}+q^{2}\left(C^{\natural}\right)^{2}-q A^{\natural} \alpha^{\natural}-q^{-1} B^{\natural} \beta^{\natural}-q C^{\natural} \gamma^{\natural},
$$

where $A^{\natural}, B^{\natural}, C^{\natural}, \alpha^{\natural}, \beta^{\natural}, \gamma^{\natural}$ are from (4.1)-(4.6). We show that (5.1) is equal to (2.18). Define $Q$ to be (5.1) minus (2.18). We show that $Q=0$. To do this we proceed as in the proof of Theorem 2.16. View $Q$ as a Laurent polynomial in $a, b, c$ that has all coefficients in $U$. We will show that for this polynomial each coefficient is zero. To this end, evaluate $Q$ using (4.1)-(4.6) and then collect terms. Below we list the terms for which the coefficient in $Q$ is potentially nonzero:

$$
\begin{aligned}
& a^{2}, \quad a^{-2}, \quad a^{2} b^{-2}, \quad a b c^{-1}, \quad a b^{-1} c^{-1}, \\
& b^{2}, \quad b^{-2}, \quad b^{2} c^{-2}, \quad b c a^{-1}, \quad b c^{-1} a^{-1}, \\
& c^{2}, \quad c^{-2}, \quad c^{2} a^{-2}, \quad c a b^{-1}, \quad c a^{-1} b^{-1}, \\
& a b c, \quad a^{-1} b^{-1} c^{-1}, \quad 1 \text {. }
\end{aligned}
$$

We show that for each term in (5.2)-(5.5) the coefficient in $Q$ is zero. The coefficient of $a^{2}$ in $Q$ is

$$
q x y \nu_{y}+q^{2} x^{2}-q x \Lambda-q \nu_{y}+1 .
$$

To see that (5.6) is zero, eliminate $x y$ using the equation on the left in (3.4), and evaluate the result using the equation on the right in (3.20). The coefficient of $a^{-2}$ in $Q$ is

$$
q y \nu_{x} x+q^{2} y^{2}-q \Lambda y-q^{-1} \nu_{x}+1 .
$$

To see that (5.7) is zero, first eliminate $y \nu_{x}$ using the equation on the right in (3.11). Evaluate the result using the equation on the right in (3.4) followed by the equation on the right in (3.21). The coefficient of $a^{2} b^{-2}$ in $Q$ is

$$
q x z \nu_{y}+q^{2} \nu_{y}^{2}-q \nu_{y} .
$$

The expression (5.8) is zero by the equation on the right in (3.6). The coefficient of $a b c^{-1}$ in $Q$ is

$$
q\left(x y x+\nu_{z} y \nu_{y}\right)+q^{2}\left(x \nu_{z}+\nu_{z} x\right)-q\left(x+\Lambda \nu_{z}\right)-q^{-1} y-q x+\Lambda .
$$

To see that (5.9) is zero, eliminate $y x$ using the equation on the right in (3.4), and eliminate $y \nu_{y}$ using the equation on the right in (3.14). Simplify the result using the equation on the left in (3.15). The coefficient of $a b^{-1} c^{-1}$ in $Q$ is

$$
q\left(x z x+\nu_{z} z \nu_{y}\right)+q^{2}\left(x \nu_{y}+\nu_{y} x\right)-q x-q^{-1} z-q\left(x+\Lambda \nu_{y}\right)+\Lambda .
$$

To see that (5.10) is zero, eliminate $x z$ using the equation on the right in (3.6), and eliminate $\nu_{z} z$ using the equation on the left in (3.15). Simplify the result using the equation on the right in (3.14). We have shown that for each term in (5.2) the coefficient in $Q$ is zero. By Lemma 2.5 $\Omega$ is fixed by the automorphism of $\Delta$ that sends $(A, B, C)$ to $(B, C, A)$. Combining this with the $\mathbb{Z}_{3}$-symmetric nature of (4.1)-(4.6), we see that for each term in (5.3), (5.4) the coefficient in $Q$ is also zero. We now consider the terms in (5.5). The coefficient of $a b c$ in $Q$ is

$$
q x y z-q x-q^{-1} y-q z+\Lambda .
$$


The expression (5.11) is zero using the left side of (2.10). The coefficient of $a^{-1} b^{-1} c^{-1}$ in $Q$ is

$$
q y z x-q y-q^{-1} z-q x+\Lambda .
$$

The expression (5.12) is zero using the left side of (2.11). The constant term in $Q$ is

$$
\begin{aligned}
& q\left(\nu_{z} z^{2}+x \nu_{x} x+y^{2} \nu_{y}+\nu_{z} \nu_{x} \nu_{y}\right)+q^{2}(x y+y x)+q^{-2}(y z+z y)+q^{2}(z x+x z) \\
& \quad-q\left(\nu_{z}+\Lambda y+\Lambda x\right)-q^{-1}\left(\nu_{x}+\Lambda z+\Lambda y\right)-q\left(\nu_{y}+\Lambda x+\Lambda z\right)-\left(q+q^{-1}\right)^{2}+\Lambda^{2}+6 .
\end{aligned}
$$

We show that this constant term is equal to zero. Using Lemma 3.3 and Lemma 3.6 we find

$$
\begin{aligned}
& \nu_{z} z^{2}=q^{-2} \nu_{x}+q^{2} \nu_{y}+\Lambda z-q-q^{-1}, \\
& x \nu_{x} x=\nu_{y}+\nu_{z}+\Lambda x-q-q^{-1}, \\
& y^{2} \nu_{y}=q^{-2} \nu_{x}+q^{2} \nu_{z}+\Lambda y-q-q^{-1} .
\end{aligned}
$$

Using the equation on the left in (3.19), followed by (5.13) and the equation on the left in (3.15), we find

$$
\nu_{z} \nu_{x} \nu_{y}=q^{-4} \nu_{x}+\nu_{y}+\nu_{z}+\Lambda x+q^{-2} \Lambda y+q^{-2} \Lambda z-q^{-1} \Lambda^{2}-q^{-1}-q^{-3} .
$$

By Lemma 3.3,

$$
\begin{aligned}
& x y+y x=2-\left(q+q^{-1}\right) \nu_{z}, \\
& y z+z y=2-\left(q+q^{-1}\right) \nu_{x}, \\
& z x+x z=2-\left(q+q^{-1}\right) \nu_{y} .
\end{aligned}
$$

Simplifying the constant term of $Q$ using (5.13)-(5.19) we find that this constant term is equal to zero. We have shown that for each term in (5.2)-(5.5) the coefficient in $Q$ is zero. Therefore $Q=0$ and the result follows.

\section{A $\mathbb{Z}$-grading of $U$}

Our next general goal is to prove Theorem 2.18. To prepare for this proof we obtain some results about $U$. In this section we discuss a certain $\mathbb{Z}$-grading of $U$. In the next section we will use this $\mathbb{Z}$-grading of $U$ to get a $\mathbb{Z}$-grading of $U \otimes \mathbb{F}\left[a^{ \pm 1}, b^{ \pm 1}, c^{ \pm 1}\right]$. The $\mathbb{Z}$-grading of $U \otimes \mathbb{F}\left[a^{ \pm 1}, b^{ \pm 1}, c^{ \pm 1}\right]$ will be used in our proof of Theorem 2.18.

Let $\mathcal{A}$ denote an $\mathbb{F}$-algebra. By a $\mathbb{Z}$-grading of $\mathcal{A}$ we mean a sequence $\left\{\mathcal{A}_{n}\right\}_{n \in \mathbb{Z}}$ consisting of subspaces of $\mathcal{A}$ such that

$$
\mathcal{A}=\sum_{n \in \mathbb{Z}} \mathcal{A}_{n} \quad \text { (direct sum) }
$$

and $\mathcal{A}_{m} \mathcal{A}_{n} \subseteq \mathcal{A}_{m+n}$ for all $m, n \in \mathbb{Z}$. Let $\left\{\mathcal{A}_{n}\right\}_{n \in \mathbb{Z}}$ denote a $\mathbb{Z}$-grading of $\mathcal{A}$. For $n \in \mathbb{Z}$ we call $\mathcal{A}_{n}$ the $n$-homogeneous component of $\mathcal{A}$. We refer to $n$ as the degree of $\mathcal{A}_{n}$. An element of $\mathcal{A}$ is said to be homogeneous with degree $n$ whenever it is contained in $\mathcal{A}_{n}$. Pick $\xi \in \mathcal{A}$ and write $\xi=\sum_{n \in \mathbb{Z}} \xi_{n}$ with $\xi_{n} \in \mathcal{A}_{n}$ for $n \in \mathbb{Z}$. We call the elements $\left\{\xi_{n}\right\}_{n \in \mathbb{Z}}$ the homogeneous components of $\xi$.

Lemma 6.1 ([10, Theorem 1.5]). The following is a basis for the $\mathbb{F}$-vector space $U$ :

$$
e^{r} k^{s} f^{t}, \quad r, t \in \mathbb{N}, \quad s \in \mathbb{Z} .
$$


For $n \in \mathbb{Z}$ let $U_{n}$ denote the subspace of $U$ spanned by those elements $e^{r} k^{s} f^{t}$ from (6.1) that satisfy $r-t=n$. By [10, Section 1.9] the sequence $\left\{U_{n}\right\}_{n \in \mathbb{Z}}$ is a $\mathbb{Z}$-grading of $U$. With respect to this $\mathbb{Z}$-grading the elements $e, k, f$ are homogeneous with degrees $1,0,-1$ respectively. Moreover the normalized Casimir element $\Lambda$ from (2.5) is homogeneous with degree 0 .

By construction, for $n \in \mathbb{Z}$ the $n$-homogeneous component $U_{n}$ has a basis consisting of the elements $e^{r} k^{s} f^{t}$ from (6.1) that satisfy $r-t=n$. There is another basis for $U_{n}$ that is better suited to our purpose; this basis involves $\Lambda$ and will be displayed shortly.

Lemma 6.2. For an integer $t \geq 0$,

$$
e^{t} f^{t}=\prod_{i=1}^{t} \frac{\Lambda-q^{1-2 i} k-q^{2 i-1} k^{-1}}{\left(q-q^{-1}\right)^{2}} .
$$

Proof. Assume $t \geq 1$; otherwise the result is trivial. Using (2.5) and $k e=q^{2} e k$,

$$
e^{t} f^{t}=e^{t-1} \text { eff } f^{t-1}=e^{t-1} \frac{\Lambda-q^{-1} k-q k^{-1}}{\left(q-q^{-1}\right)^{2}} f^{t-1}=\frac{\Lambda-q^{1-2 t} k-q^{2 t-1} k^{-1}}{\left(q-q^{-1}\right)^{2}} e^{t-1} f^{t-1} .
$$

The result follows by induction on $t$.

Lemma 6.3. For all integers $n \geq 0$ the following $(i)$, (ii) hold.

(i) The $\mathbb{F}$-vector space $U_{n}$ has a basis

$$
e^{n} k^{s} \Lambda^{t}, \quad s \in \mathbb{Z}, \quad t \in \mathbb{N} \text {. }
$$

(ii) The $\mathbb{F}$-vector space $U_{-n}$ has a basis

$$
k^{s} \Lambda^{t} f^{n}, \quad s \in \mathbb{Z}, \quad t \in \mathbb{N} \text {. }
$$

Proof. (i) The elements $\left\{k^{s}\right\}_{s \in \mathbb{Z}}$ are linearly independent by Lemma 6.1, so they form a basis for a subalgebra of $U$ which we denote by $K$. By Lemma 6.1 the sum $U_{n}=\sum_{\ell=0}^{\infty} e^{n+\ell} K f^{\ell}$ is direct. We have $K e=e K$ since $k e=q^{2} e k$, and similarly $K f=f K$. Pick an integer $t \geq 0$. By Lemma 6.2 and induction on $t$ we find $\Lambda^{t} \in \sum_{\ell=0}^{t} e^{\ell} K f^{\ell}$ and $\Lambda^{t}-\left(q-q^{-1}\right)^{2 t} e^{t} f^{t} \in \sum_{\ell=0}^{t-1} e^{\ell} K f^{\ell}$. For the above $t$ and all $s \in \mathbb{Z}$ we have $e^{n} k^{s} \Lambda^{t} \in \sum_{\ell=0}^{t} e^{n+\ell} K f^{\ell}$ and

$$
e^{n} k^{s} \Lambda^{t}-\left(q-q^{-1}\right)^{2 t} q^{2 s t} e^{n+t} k^{s} f^{t} \in \sum_{\ell=0}^{t-1} e^{n+\ell} K f^{\ell} .
$$

The result follows from these comments and the fact that $\left\{e^{n+t} k^{s} f^{t} \mid s \in \mathbb{Z}, t \in \mathbb{N}\right\}$ is a basis for $U_{n}$.

(ii). Similar to the proof of $(i)$ above.

We now consider the $\mathbb{Z}$-grading $\left\{U_{n}\right\}_{n \in \mathbb{Z}}$ from the point of view of the equitable presentation.

Lemma 6.4. The $\mathbb{F}$-algebra $U$ is generated by $\nu_{x}, y^{ \pm 1}, \nu_{z}$. Moreover

$$
x=y^{-1}-q^{-1} \nu_{z} y^{-1}, \quad z=y^{-1}-q^{-1} y^{-1} \nu_{x} .
$$


Proof. The equation on the left in (6.2) is a reformulation of the equation on the left in (3.4). The equation on the right in (6.2) is similarly obtained. The first assertion of the lemma follows from (6.2) and the fact that $x, y^{ \pm 1}, z$ generate $U$.

Lemma 6.5. The generators $\nu_{x}, y^{ \pm 1}, \nu_{z}$ are homogeneous with degree $-1,0,1$ respectively.

Proof. Use Note 3.2 and $y=k$, along with the comments below Lemma 6.1.

Lemma 6.6. Pick an integer $n \geq 0$. The $\mathbb{F}$-vector space $U_{n}$ has a basis

$$
\nu_{z}^{n} y^{i} \Lambda^{j}, \quad i \in \mathbb{Z}, \quad j \in \mathbb{N} .
$$

The $\mathbb{F}$-vector space $U_{-n}$ has a basis

$$
y^{i} \Lambda^{j} \nu_{x}^{n}, \quad i \in \mathbb{Z}, \quad j \in \mathbb{N} .
$$

Proof. This is a reformulation of Lemma 6.3, using Note 3.2 and $y=k$.

We comment on the homogeneous component $U_{0}$.

Lemma 6.7. The homogeneous component $U_{0}$ is the $\mathbb{F}$-subalgebra of $U$ generated by $y^{ \pm 1}, \Lambda$. The algebra $U_{0}$ is commutative. The following is a basis for the $\mathbb{F}$-vector space $U_{0}$ :

$$
y^{i} \Lambda^{j}, \quad i \in \mathbb{Z}, \quad j \in \mathbb{N} .
$$

Proof. To get the basis (6.3) set $n=0$ in Lemma 6.6. The remaining assertions are clear.

Let $\lambda_{1}, \lambda_{2}$ denote commuting indeterminates.

Corollary 6.8. There exists an $\mathbb{F}$-algebra isomorphism $U_{0} \rightarrow \mathbb{F}\left[\lambda_{1}, \lambda_{2}^{ \pm 1}\right]$ that sends $\Lambda \rightarrow \lambda_{1}$ and $y \rightarrow \lambda_{2}$.

Proof. Immediate from Lemma 6.7.

Definition 6.9. For $n \in \mathbb{Z}$ define an $\mathbb{F}$-linear map $\pi_{n}: U \rightarrow U$ such that $\left(\pi_{n}-1\right) U_{n}=0$ and $\pi_{n} U_{m}=0$ if $m \neq n(m \in \mathbb{Z})$. Thus $\pi_{n}$ is the projection from $U$ onto $U_{n}$. Note that for $u \in U$ the element $\pi_{n}(u)$ is the homogeneous component of $u$ with degree $n$.

Lemma 6.10. In the table below we list some elements $u \in U$. For each element $u$ we display the homogeneous component $\pi_{n}(u)$ for $-1 \leq n \leq 1$. All other homogeneous components of $u$ are zero.

\begin{tabular}{c|ccc}
$u$ & $\pi_{-1}(u)$ & $\pi_{0}(u)$ & $\pi_{1}(u)$ \\
\hline \hline$x$ & 0 & $y^{-1}$ & $-q^{-1} \nu_{z} y^{-1}$ \\
$y$ & 0 & $y$ & 0 \\
$z$ & $-q^{-1} y^{-1} \nu_{x}$ & $y^{-1}$ & 0 \\
\hline$\nu_{x}$ & $\nu_{x}$ & 0 & 0 \\
$\nu_{y}$ & $q^{-2} y^{-2} \nu_{x}$ & $y^{-1} \Lambda-\left(q+q^{-1}\right) y^{-2}$ & $q^{-2} \nu_{z} y^{-2}$ \\
$\nu_{z}$ & 0 & 0 & $\nu_{z}$ \\
\hline$\Lambda$ & 0 & $\Lambda$ & 0
\end{tabular}

Proof. The assertions about $y, \nu_{x}, \nu_{z}$ come from Lemma 6.5. The assertions about $x, z$ follow from (6.2). We mentioned below Lemma 6.1 that $\Lambda$ is homogeneous with degree 0 . To verify the assertion about $\nu_{y}$, in the equation $\nu_{y}=y^{-1} y \nu_{y}$ evaluate $y \nu_{y}$ using the equation on the right in (3.14), and simplify the result using rows $x, z$ of the above table along with the equation on the left in (3.11). 


\section{$7 \quad$ A $\mathbb{Z}$-grading of $U \otimes \mathbb{F}\left[a^{ \pm 1}, b^{ \pm 1}, c^{ \pm 1}\right]$}

In the previous section we discussed a $\mathbb{Z}$-grading of $U$. In the present section we extend this to a $\mathbb{Z}$-grading of $U \otimes \mathbb{F}\left[a^{ \pm 1}, b^{ \pm 1}, c^{ \pm 1}\right]$. We obtain some results about the $\mathbb{Z}$-grading of $U \otimes$ $\mathbb{F}\left[a^{ \pm 1}, b^{ \pm 1}, c^{ \pm 1}\right]$ that will be used to prove Theorem 2.18.

By Lemma 6.4 the elements $\nu_{x}, y^{ \pm 1}, \nu_{z}$ form a generating set for the $\mathbb{F}$-algebra $U$. Therefore the following is a generating set for the $\mathbb{F}$-algebra $U \otimes \mathbb{F}\left[a^{ \pm 1}, b^{ \pm 1}, c^{ \pm 1}\right]$ :

$$
\nu_{x} \otimes 1, \quad y^{ \pm 1} \otimes 1, \quad \nu_{z} \otimes 1, \quad 1 \otimes a^{ \pm 1}, \quad 1 \otimes b^{ \pm 1}, \quad 1 \otimes c^{ \pm 1} .
$$

Consider the $\mathbb{Z}$-grading of $U$ from below Lemma 6.1 . This $\mathbb{Z}$-grading of $U$ induces a $\mathbb{Z}$-grading of $U \otimes \mathbb{F}\left[a^{ \pm 1}, b^{ \pm 1}, c^{ \pm 1}\right]$ whose homogeneous components are described as follows. For $n \in \mathbb{Z}$ the $n$-homogeneous component is $U_{n} \otimes \mathbb{F}\left[a^{ \pm 1}, b^{ \pm 1}, c^{ \pm 1}\right]$. With respect to this $\mathbb{Z}$-grading of $U \otimes$ $\mathbb{F}\left[a^{ \pm 1}, b^{ \pm 1}, c^{ \pm 1}\right]$ the generators (7.1) are homogeneous with the following degrees:

$$
\begin{array}{c||ccc|ccc}
v & \nu_{x} \otimes 1 & y^{ \pm 1} \otimes 1 & \nu_{z} \otimes 1 & 1 \otimes a^{ \pm 1} & 1 \otimes b^{ \pm 1} & 1 \otimes c^{ \pm 1} \\
\hline \text { degree of } v & -1 & 0 & 1 & 0 & 0 & 0
\end{array}
$$

Lemma 7.1. Pick an integer $n \geq 0$. The $\mathbb{F}$-vector space $U_{n} \otimes \mathbb{F}\left[a^{ \pm 1}, b^{ \pm 1}, c^{ \pm 1}\right]$ has a basis

$$
\nu_{z}^{n} y^{i} \Lambda^{j} \otimes a^{r} b^{s} c^{t}, \quad i, r, s, t \in \mathbb{Z}, \quad j \in \mathbb{N} .
$$

The $\mathbb{F}$-vector space $U_{-n} \otimes \mathbb{F}\left[a^{ \pm 1}, b^{ \pm 1}, c^{ \pm 1}\right]$ has a basis

$$
y^{i} \Lambda^{j} \nu_{x}^{n} \otimes a^{r} b^{s} c^{t}, \quad i, r, s, t \in \mathbb{Z}, \quad j \in \mathbb{N} .
$$

Proof. By Lemma 6.6 and the construction.

We comment on the homogeneous component $U_{0} \otimes \mathbb{F}\left[a^{ \pm 1}, b^{ \pm 1}, c^{ \pm 1}\right]$.

Lemma 7.2. The homogeneous component $U_{0} \otimes \mathbb{F}\left[a^{ \pm 1}, b^{ \pm 1}, c^{ \pm 1}\right]$ is the $\mathbb{F}$-subalgebra of $U \otimes$ $\mathbb{F}\left[a^{ \pm 1}, b^{ \pm 1}, c^{ \pm 1}\right]$ generated by

$$
y^{ \pm 1} \otimes 1, \quad \Lambda \otimes 1, \quad 1 \otimes a^{ \pm 1}, \quad 1 \otimes b^{ \pm 1}, \quad 1 \otimes c^{ \pm 1} .
$$

The algebra $U_{0} \otimes \mathbb{F}\left[a^{ \pm 1}, b^{ \pm 1}, c^{ \pm 1}\right]$ is commutative. The following is a basis for the $\mathbb{F}$-vector space $U_{0} \otimes \mathbb{F}\left[a^{ \pm 1}, b^{ \pm 1}, c^{ \pm 1}\right]:$

$$
y^{i} \Lambda^{j} \otimes a^{r} b^{s} c^{t}, \quad i, r, s, t \in \mathbb{Z}, \quad j \in \mathbb{N} .
$$

Proof. By Lemma 6.7 and the construction.

Let $\left\{\lambda_{i}\right\}_{i=1}^{5}$ denote mutually commuting indeterminates.

Corollary 7.3. There exists an $\mathbb{F}$-algebra isomorphism

$$
U_{0} \otimes \mathbb{F}\left[a^{ \pm 1}, b^{ \pm 1}, c^{ \pm 1}\right] \rightarrow \mathbb{F}\left[\lambda_{1}, \lambda_{2}^{ \pm 1}, \lambda_{3}^{ \pm 1}, \lambda_{4}^{ \pm 1}, \lambda_{5}^{ \pm 1}\right]
$$

that sends

$$
\Lambda \otimes 1 \mapsto \lambda_{1}, \quad y \otimes 1 \mapsto \lambda_{2}, \quad 1 \otimes a \mapsto \lambda_{3}, \quad 1 \otimes b \mapsto \lambda_{4}, \quad 1 \otimes c \mapsto \lambda_{5}
$$

Proof. Immediate from Lemma 7.2. 
Definition 7.4. For $n \in \mathbb{Z}$ consider the map

$$
\begin{array}{rlrl}
\pi_{n} \otimes 1: \quad U \otimes \mathbb{F}\left[a^{ \pm 1}, b^{ \pm 1}, c^{ \pm 1}\right] & \rightarrow U \otimes \mathbb{F}\left[a^{ \pm 1}, b^{ \pm 1}, c^{ \pm 1}\right] \\
u \otimes f & \mapsto & \pi_{n}(u) \otimes f .
\end{array}
$$

The map $\pi_{n} \otimes 1$ acts as the identity on the $n$-homogeneous component of $U \otimes \mathbb{F}\left[a^{ \pm 1}, b^{ \pm 1}, c^{ \pm 1}\right]$ and zero on all other homogeneous components of $U \otimes \mathbb{F}\left[a^{ \pm 1}, b^{ \pm 1}, c^{ \pm 1}\right]$. Therefore $\pi_{n} \otimes 1$ is the projection from $U \otimes \mathbb{F}\left[a^{ \pm 1}, b^{ \pm 1}, c^{ \pm 1}\right]$ onto its $n$-homogeneous component. We abbreviate $\tilde{\pi}_{n}=\pi_{n} \otimes 1$. So for $v \in U \otimes \mathbb{F}\left[a^{ \pm 1}, b^{ \pm 1}, c^{ \pm 1}\right]$ the element $\tilde{\pi}_{n}(v)$ is the homogeneous component of $v$ that has degree $n$.

Lemma 7.5. In the table below we list some elements $v \in U \otimes \mathbb{F}\left[a^{ \pm 1}, b^{ \pm 1}, c^{ \pm 1}\right]$. For each element $v$ we display the homogeneous component $\tilde{\pi}_{n}(v)$ for $-1 \leq n \leq 1$. All other homogeneous components of $v$ are zero.

\begin{tabular}{c|ccc}
$v$ & $\tilde{\pi}_{-1}(v)$ & $\tilde{\pi}_{0}(v)$ & $\tilde{\pi}_{1}(v)$ \\
\hline \hline$A^{\natural}$ & 0 & $y \otimes a^{-1}+y^{-1} \otimes a$ & $\nu_{z} \otimes b c^{-1}-q^{-1} \nu_{z} y^{-1} \otimes a$ \\
$B^{\natural}$ & $\nu_{x} \otimes a^{-1} c-q^{-1} y^{-1} \nu_{x} \otimes b^{-1}$ & $y \otimes b+y^{-1} \otimes b^{-1}$ & 0 \\
$C^{\natural}$ & $q^{-2} y^{-2} \nu_{x} \otimes a b^{-1}$ & $y^{-1} \otimes\left(c+c^{-1}\right)+y^{-1} \Lambda \otimes a b^{-1}$ & $q^{-2} \nu_{z} y^{-2} \otimes a b^{-1}$ \\
& $-q^{-1} y^{-1} \nu_{x} \otimes c$ & $-\left(q+q^{-1}\right) y^{-2} \otimes a b^{-1}$ & $-q^{-1} \nu_{z} y^{-1} \otimes c^{-1}$
\end{tabular}

Moreover each of $\alpha^{\natural}, \beta^{\natural}, \gamma^{\natural}, \Omega^{\natural}$ is homogeneous with degree zero.

Proof. The elements $A^{\natural}, B^{\natural}, C^{\natural}$ are from (4.1)-(4.3) and $\alpha^{\natural}, \beta^{\natural}, \gamma^{\natural}$ are from (4.4)-(4.6). Moreover $\Omega^{\natural}$ is from (2.18). Evaluate these lines using Lemma 6.10 and the fact that each of $1 \otimes a^{ \pm 1}, 1 \otimes b^{ \pm 1}, 1 \otimes c^{ \pm 1}$ is homogeneous of degree 0 .

The following definition is for notational convenience.

Definition 7.6. Define $R$ and $L$ by

$$
\begin{aligned}
& R=\tilde{\pi}_{1}\left(A^{\natural}\right)=\nu_{z} \otimes b c^{-1}-q^{-1} \nu_{z} y^{-1} \otimes a, \\
& L=\tilde{\pi}_{-1}\left(B^{\natural}\right)=\nu_{x} \otimes a^{-1} c-q^{-1} y^{-1} \nu_{x} \otimes b^{-1} .
\end{aligned}
$$

Further define

$$
\theta=y^{-1} \otimes a, \quad \vartheta=y^{-1} \otimes b^{-1} .
$$

Lemma 7.7. The elements $R, L, \theta, \vartheta$ from Definition 7.6 are all nonzero. Moreover

$$
\begin{array}{ll}
R \vartheta=q^{2} \vartheta R, & L \theta=q^{-2} \theta L, \\
\tilde{\pi}_{0}\left(A^{\natural}\right)=\theta+\theta^{-1}, & \tilde{\pi}_{0}\left(B^{\natural}\right)=\vartheta+\vartheta^{-1}, \\
\tilde{\pi}_{1}\left(C^{\natural}\right)=-q^{-1} R \vartheta, & \tilde{\pi}_{-1}\left(C^{\natural}\right)=-q^{-1} \theta L .
\end{array}
$$

Proof. The first assertion follows from Lemma 7.1. (7.3) follows from (3.11) and Definition 7.6. (7.4), (7.5) are readily checked using the table in Lemma 7.5.

We now give two lemmas of a slightly technical nature.

Lemma 7.8. For an integer $i \geq 0$ the homogeneous components of $\left(A^{\natural}\right)^{i},\left(B^{\natural}\right)^{i},\left(C^{\natural}\right)^{i}$ are described as follows.

$\left(A^{\natural}\right)^{i}$ : The homogeneous component of degree $n$ is zero unless $0 \leq n \leq i$. The homogeneous component of degree 0 is $\left(\theta+\theta^{-1}\right)^{i}$ and the homogeneous component of degree $i$ is $R^{i}$. 
$\left(B^{\natural}\right)^{i}$ : The homogeneous component of degree $n$ is zero unless $-i \leq n \leq 0$. The homogeneous component of degree $-i$ is $L^{i}$ and the homogeneous component of degree 0 is $\left(\vartheta+\vartheta^{-1}\right)^{i}$.

$\left(C^{\natural}\right)^{i}$ : The homogeneous component of degree $n$ is zero unless $-i \leq n \leq i$. The homogeneous component of degree $-i$ is $(-1)^{i} q^{i^{2}} L^{i} \theta^{i}$ and the homogeneous component of degree $i$ is $(-1)^{i} q^{-i^{2}} R^{i} \vartheta^{i}$

Proof. This is readily checked using Lemma 7.5 and Lemma 7.7.

Using Lemma 7.7 and Lemma 7.8 we routinely obtain the following result.

Lemma 7.9. Fix nonnegative integers $i, j, k$ and consider the homogeneous components of $\left(A^{\natural}\right)^{i}\left(B^{\natural}\right)^{j}\left(C^{\natural}\right)^{k}$. The homogeneous component of degree $n$ is zero unless $-j-k \leq n \leq i+k$. The homogeneous component of degree $-j-k$ is

$$
(-1)^{k} q^{k^{2}} L^{j+k}\left(q^{2 j+2 k} \theta+q^{-2 j-2 k} \theta^{-1}\right)^{i} \theta^{k} .
$$

The homogeneous component of degree $i+k$ is

$$
(-1)^{k} q^{-k^{2}} R^{i+k}\left(q^{-2 k} \vartheta+q^{2 k} \vartheta^{-1}\right)^{j} \vartheta^{k} .
$$

\section{Some results concerning algebraic independence}

In this section we establish some results about algebraic independence that will be used in the proof of Theorem 2.18.

Let $\left\{x_{i}\right\}_{i=1}^{4}$ denote mutually commuting indeterminates. Motivated by the form of (2.18) and (4.4)-(4.6) we consider the following elements in $\mathbb{F}\left[x_{1}, x_{2}, x_{3}, x_{4}\right]$ :

$$
\begin{aligned}
& y_{1}=x_{1} x_{2} x_{3} x_{4}+x_{1}^{2}+x_{2}^{2}+x_{3}^{2}+x_{4}^{2}, \\
& y_{2}=x_{1} x_{2}+x_{3} x_{4}, \quad y_{3}=x_{1} x_{3}+x_{2} x_{4}, \quad y_{4}=x_{1} x_{4}+x_{2} x_{3} .
\end{aligned}
$$

Lemma 8.1. The elements $\left\{y_{i}\right\}_{i=1}^{4}$ in (8.1), (8.2) are algebraically independent over $\mathbb{F}$.

Proof. The following is a basis for the $\mathbb{F}$-vector space $\mathbb{F}\left[x_{1}, x_{2}, x_{3}, x_{4}\right]$ :

$$
x_{1}^{h} x_{2}^{i} x_{3}^{j} x_{4}^{k}, \quad h, i, j, k \in \mathbb{N} .
$$

An element $x_{1}^{h} x_{2}^{i} x_{3}^{j} x_{4}^{k}$ in the basis (8.3) will be called a monomial. The rank of this monomial is defined to be $2 h+i+j+k$. For example, consider the monomials that make up $y_{1}$ in (8.1). The monomial $x_{1} x_{2} x_{3} x_{4}$ has rank 5 , and the remaining monomials $x_{1}^{2}, x_{2}^{2}, x_{3}^{2}, x_{4}^{2}$ have rank 4 , 2, 2, 2 respectively. For $2 \leq i \leq 4$ consider the two monomials that make up $y_{i}$ in (8.2). In each case the monomial involving $x_{1}$ has rank 3 and the other monomial has rank 2 . To prove the lemma, it suffices to show that the following elements are linearly independent over $\mathbb{F}$ :

$$
y_{1}^{r} y_{2}^{s} y_{3}^{t} y_{4}^{u}, \quad r, s, t, u \in \mathbb{N} .
$$

Given integers $r, s, t, u \geq 0$ write $y_{1}^{r} y_{2}^{s} y_{3}^{t} y_{4}^{u}$ as a linear combination of monomials:

$$
\begin{aligned}
y_{1}^{r} y_{2}^{s} y_{3}^{t} y_{4}^{u} & =\left(x_{1} x_{2} x_{3} x_{4}+x_{1}^{2}+x_{2}^{2}+x_{3}^{2}+x_{4}^{2}\right)^{r}\left(x_{1} x_{2}+x_{3} x_{4}\right)^{s}\left(x_{1} x_{3}+x_{2} x_{4}\right)^{t}\left(x_{1} x_{4}+x_{2} x_{3}\right)^{u} \\
& =\left(x_{1} x_{2} x_{3} x_{4}\right)^{r}\left(x_{1} x_{2}\right)^{s}\left(x_{1} x_{3}\right)^{t}\left(x_{1} x_{4}\right)^{u}+\text { sum of monomials that have lower rank } \\
& =x_{1}^{r+s+t+u} x_{2}^{r+s} x_{3}^{r+t} x_{4}^{r+u}+\text { sum of monomials that have lower rank. }
\end{aligned}
$$


Let us call the monomial $x_{1}^{r+s+t+u} x_{2}^{r+s} x_{3}^{r+t} x_{4}^{r+u}$ the leading monomial for $y_{1}^{r} y_{2}^{s} y_{3}^{t} y_{4}^{u}$. Given a monomial $x_{1}^{h} x_{2}^{i} x_{3}^{j} x_{4}^{k}$ in the basis (8.3), consider the following system of linear equations in the unknowns $r, s, t, u$ :

$$
r+s+t+u=h, \quad r+s=i, \quad r+t=j, \quad r+u=k .
$$

Over the rational field $\mathbb{Q}$ this system has a unique solution

$$
r=\frac{i+j+k-h}{2}, \quad s=\frac{h+i-j-k}{2}, \quad t=\frac{h-i+j-k}{2}, \quad u=\frac{h-i-j+k}{2} .
$$

Therefore $x_{1}^{h} x_{2}^{i} x_{3}^{j} x_{4}^{k}$ is the leading monomial for at most one element of (8.4). By these comments the elements (8.4) are linearly independent over $\mathbb{F}$. The result follows.

Recall the commutative algebra $U_{0} \otimes \mathbb{F}\left[a^{ \pm 1}, b^{ \pm 1}, c^{ \pm 1}\right]$ from Lemma 7.2, and the element $\theta=$ $y^{-1} \otimes a$ from (7.2).

Proposition 8.2. The following elements of $U_{0} \otimes \mathbb{F}\left[a^{ \pm 1}, b^{ \pm 1}, c^{ \pm 1}\right]$ are algebraically independent over $\mathbb{F}$ :

$$
\theta, \quad \Omega^{\natural}, \quad \alpha^{\natural}, \quad \beta^{\natural}, \quad \gamma^{\natural} .
$$

Proof. By Corollary 7.3 the following are algebraically independent over $\mathbb{F}$ :

$$
y \otimes 1, \quad \Lambda \otimes 1, \quad 1 \otimes a, \quad 1 \otimes b, \quad 1 \otimes c .
$$

Therefore the following are algebraically independent over $\mathbb{F}$ :

$$
y^{-1} \otimes a, \quad \Lambda \otimes 1, \quad 1 \otimes a, \quad 1 \otimes b, \quad 1 \otimes c .
$$

Therefore the following are algebraically independent over $\mathbb{F}$ :

$$
y^{-1} \otimes a, \quad \Lambda \otimes 1, \quad 1 \otimes\left(a+a^{-1}\right), \quad 1 \otimes\left(b+b^{-1}\right), \quad 1 \otimes\left(c+c^{-1}\right) .
$$

Abbreviate the sequence (8.5) by $X_{0}, X_{1}, X_{2}, X_{3}, X_{4}$. By Lemma 8.1 the following are algebraically independent over $\mathbb{F}$ :

$$
\begin{aligned}
& X_{0}, \quad X_{1} X_{2} X_{3} X_{4}+X_{1}^{2}+X_{2}^{2}+X_{3}^{2}+X_{4}^{2}, \\
& X_{1} X_{2}+X_{3} X_{4}, \quad X_{1} X_{3}+X_{2} X_{4}, \quad X_{1} X_{4}+X_{2} X_{3} .
\end{aligned}
$$

The above five elements are

$$
\theta, \quad 1 \otimes\left(q+q^{-1}\right)^{2}-\Omega^{\natural}, \quad \alpha^{\natural}, \quad \beta^{\natural}, \quad \gamma^{\natural},
$$

respectively. The result follows.

Recall the element $\vartheta=y^{-1} \otimes b^{-1}$ from (7.2).

Proposition 8.3. The following elements of $U_{0} \otimes \mathbb{F}\left[a^{ \pm 1}, b^{ \pm 1}, c^{ \pm 1}\right]$ are algebraically independent over $\mathbb{F}$ :

$$
\vartheta, \quad \Omega^{\natural}, \quad \alpha^{\natural}, \quad \beta^{\natural}, \quad \gamma^{\natural} .
$$

Proof. Similar to the proof of Proposition 8.2. 


\section{The proof of Theorem 2.18}

In this section we prove Theorem 2.18 .

Lemma 9.1 ([17, Theorem 7.5]). The following is a basis for the $\mathbb{F}$-vector space $\Delta$ :

$$
A^{i} B^{j} C^{k} \Omega^{\ell} \alpha^{r} \beta^{s} \gamma^{t}, \quad i, j, k, \ell, r, s, t \in \mathbb{N}, \quad i j k=0 .
$$

We will be discussing the coefficients when an element of $\Delta$ is written as a linear combination of the basis elements (9.1). To facilitate this discussion we define a bilinear form $\langle\rangle:, \Delta \times \Delta \rightarrow \mathbb{F}$ such that $\langle u, v\rangle=\delta_{u, v}$ for all elements $u, v$ in the basis (9.1). The bilinear form $\langle$,$\rangle is symmetric,$ and the basis (9.1) is orthonormal with respect to $\langle$,$\rangle . For v \in \Delta$,

$$
v=\sum\left\langle v, A^{i} B^{j} C^{k} \Omega^{\ell} \alpha^{r} \beta^{s} \gamma^{t}\right\rangle A^{i} B^{j} C^{k} \Omega^{\ell} \alpha^{r} \beta^{s} \gamma^{t},
$$

where the sum is over all elements $A^{i} B^{j} C^{k} \Omega^{\ell} \alpha^{r} \beta^{s} \gamma^{t}$ in the basis (9.1). In this sum there are finitely many nonzero summands.

Proof of Theorem 2.18. Let $J \subseteq \Delta$ denote the kernel of $\natural$. We show that $J=0$. To do this we assume $J \neq 0$ and get a contradiction. Fix $0 \neq v \in J$. Let $S=S(v)$ denote the set of 7-tuples $(i, j, k, \ell, r, s, t)$ of nonnegative integers such that $i j k=0$ and $\left\langle v, A^{i} B^{j} C^{k} \Omega^{\ell} \alpha^{r} \beta^{s} \gamma^{t}\right\rangle \neq 0$. By construction

$$
v=\sum_{(i, j, k, \ell, r, s, t) \in S}\left\langle v, A^{i} B^{j} C^{k} \Omega^{\ell} \alpha^{r} \beta^{s} \gamma^{t}\right\rangle A^{i} B^{j} C^{k} \Omega^{\ell} \alpha^{r} \beta^{s} \gamma^{t} .
$$

In this equation we apply $\curvearrowleft$ to both sides and get

$$
0=\sum_{(i, j, k, \ell, r, s, t) \in S}\left\langle v, A^{i} B^{j} C^{k} \Omega^{\ell} \alpha^{r} \beta^{s} \gamma^{t}\right\rangle\left(A^{\natural}\right)^{i}\left(B^{\natural}\right)^{j}\left(C^{\natural}\right)^{k}\left(\Omega^{\natural}\right)^{\ell}\left(\alpha^{\natural}\right)^{r}\left(\beta^{\natural}\right)^{s}\left(\gamma^{\natural}\right)^{t} .
$$

For an element $(i, j, k, \ell, r, s, t) \in S$ define its height to be $i+k$ and its depth to be $j+k$. For all integers $n \geq 0$ let $S_{n}^{+}$(resp. $S_{n}^{-}$) denote the set of elements in $S$ that have height $n$ (resp. depth $n)$. By construction $\left\{S_{n}^{+}\right\}_{n=0}^{\infty}$ (resp. $\left.\left\{S_{n}^{-}\right\}_{n=0}^{\infty}\right)$ is a partition of $S$. We assume $v \neq 0$ so $S$ is nonempty. Therefore $\left\{S_{n}^{+}\right\}_{n=0}^{\infty}$ (resp. $\left\{S_{n}^{-}\right\}_{n=0}^{\infty}$ ) are not all empty. By construction $S$ has finite cardinality, so finitely many of $\left\{S_{n}^{+}\right\}_{n=0}^{\infty}$ (resp. $\left\{S_{n}^{-}\right\}_{n=0}^{\infty}$ ) are nonempty. Define $N=\max \left\{n \mid S_{n}^{+} \neq \varnothing\right\}$ and $M=\max \left\{n \mid S_{n}^{-} \neq \varnothing\right\}$. By construction $S_{N}^{+}$and $S_{M}^{-}$are nonempty. We now split the argument into two cases.

Case $N \leq M$ : Recall the projection map $\tilde{\pi}_{-M}$ from Definition 7.4. Apply $\tilde{\pi}_{-M}$ to each side of (9.2). Pick $(i, j, k, \ell, r, s, t) \in S$ and consider the corresponding summand in (9.2). The image of this summand under $\tilde{\pi}_{-M}$ is computed using Lemma 7.9, and found to be zero unless $(i, j, k, \ell, r, s, t) \in S_{M}^{-}$. The result of the computation is that

$$
0=L^{M} \sum_{(i, j, k, \ell, r, s, t) \in S_{M}^{-}}\left\langle v, A^{i} B^{j} C^{k} \Omega^{\ell} \alpha^{r} \beta^{s} \gamma^{t}\right\rangle(-1)^{k} q^{k^{2}}\left(q^{2 M} \theta+q^{-2 M} \theta^{-1}\right)^{i} \theta^{k}\left(\Omega^{\natural}\right)^{\ell}\left(\alpha^{\natural}\right)^{r}\left(\beta^{\natural}\right)^{s}\left(\gamma^{\natural}\right)^{t},
$$

where $L, \theta$ are from Definition 7.6. By Lemma 7.7 $L \neq 0$. We mentioned above Corollary 2.19 that $U \otimes \mathbb{F}\left[a^{ \pm 1}, b^{ \pm 1}, c^{ \pm 1}\right]$ contains no zero divisors. Therefore

$$
\begin{gathered}
0=\sum_{(i, j, k, \ell, r, s, t) \in S_{M}^{-}}\left\langle v, A^{i} B^{j} C^{k} \Omega^{\ell} \alpha^{r} \beta^{s} \gamma^{t}\right\rangle(-1)^{k} q^{k^{2}}\left(q^{2 M} \theta+q^{-2 M} \theta^{-1}\right)^{i} \theta^{k} \\
\times\left(\Omega^{\natural}\right)^{\ell}\left(\alpha^{\natural}\right)^{r}\left(\beta^{\natural}\right)^{s}\left(\gamma^{\natural}\right)^{t} .
\end{gathered}
$$


Consider the above equation. We noted earlier that $S_{M}^{-}$is nonempty. By construction the scalar $\left\langle v, A^{i} B^{j} C^{k} \Omega^{\ell} \alpha^{r} \beta^{s} \gamma^{t}\right\rangle$ is nonzero for all $(i, j, k, \ell, r, s, t) \in S_{M}^{-}$. For $(i, j, k, \ell, r, s, t) \in S_{M}^{-}$, at least one of $i, j, k$ is zero since $i j k=0$. Moreover $j+k=M$ and $i+k \leq M$. For these constraints on $i, j, k$ the possible solutions for $(i, j, k)$ are

$$
(0,0, M),(0,1, M-1), \ldots,(0, M-1,1),(0, M, 0),(1, M, 0), \ldots,(M-1, M, 0),(M, M, 0) .
$$

For the above values of $(i, j, k)$ the corresponding values of $\left(q^{2 M} \theta+q^{-2 M} \theta^{-1}\right)^{i} \theta^{k}$ are

$$
\theta^{M}, \theta^{M-1}, \ldots, \theta, 1, q^{2 M} \theta+q^{-2 M} \theta^{-1}, \ldots,\left(q^{2 M} \theta+q^{-2 M} \theta^{-1}\right)^{M-1},\left(q^{2 M} \theta+q^{-2 M} \theta^{-1}\right)^{M} .
$$

The above line contains a sequence of Laurent polynomials in $\theta$. (9.4) below contains a sequence of Laurent polynomials in $\theta$. These two sequences are bases for the same vector space.

$$
\theta^{M}, \theta^{M-1}, \ldots, \theta, 1, \theta^{-1}, \ldots, \theta^{1-M}, \theta^{-M} .
$$

With the above comments in mind, equation (9.3) gives a nontrivial $\mathbb{F}$-linear dependency among

$$
\theta^{h}\left(\Omega^{\natural}\right)^{\ell}\left(\alpha^{\natural}\right)^{r}\left(\beta^{\natural}\right)^{s}\left(\gamma^{\natural}\right)^{t}, \quad \ell, r, s, t \in \mathbb{N}, \quad h \in \mathbb{Z}, \quad-M \leq h \leq M .
$$

In the above line we multiply each term by $\theta^{M}$ and obtain a nontrivial $\mathbb{F}$-linear dependency among

$$
\theta^{h}\left(\Omega^{\natural}\right)^{\ell}\left(\alpha^{\natural}\right)^{r}\left(\beta^{\natural}\right)^{s}\left(\gamma^{\natural}\right)^{t}, \quad h, \ell, r, s, t \in \mathbb{N}, \quad h \leq 2 M .
$$

The above linear dependency contradicts Proposition 8.2, for the present case $N \leq M$.

Case $M \leq N$ : The argument is similar to the previous case. However the details are slightly different so we will show them. Apply $\tilde{\pi}_{N}$ to each side of (9.2). Pick $(i, j, k, \ell, r, s, t) \in S$ and consider the corresponding summand in (9.2). The image of this summand under $\tilde{\pi}_{N}$ is computed using Lemma 7.9, and found to be zero unless $(i, j, k, \ell, r, s, t) \in S_{N}^{+}$. The result of the computation is that

$$
\begin{gathered}
0=R^{N} \sum_{(i, j, k, \ell, r, s, t) \in S_{N}^{+}}\left\langle v, A^{i} B^{j} C^{k} \Omega^{\ell} \alpha^{r} \beta^{s} \gamma^{t}\right\rangle(-1)^{k} q^{-k^{2}}\left(q^{-2 k} \vartheta+q^{2 k} \vartheta^{-1}\right)^{j} \vartheta^{k} \\
\times\left(\Omega^{\natural}\right)^{\ell}\left(\alpha^{\natural}\right)^{r}\left(\beta^{\natural}\right)^{s}\left(\gamma^{\natural}\right)^{t},
\end{gathered}
$$

where $R, \vartheta$ are from Definition 7.6. By Lemma $7.7 R \neq 0$. We have seen that $U \otimes \mathbb{F}\left[a^{ \pm 1}, b^{ \pm 1}, c^{ \pm 1}\right]$ contains no zero divisors. Therefore

$$
\begin{gathered}
0=\sum_{(i, j, k, \ell, r, s, t) \in S_{N}^{+}}\left\langle v, A^{i} B^{j} C^{k} \Omega^{\ell} \alpha^{r} \beta^{s} \gamma^{t}\right\rangle(-1)^{k} q^{-k^{2}}\left(q^{-2 k} \vartheta+q^{2 k} \vartheta^{-1}\right)^{j} \vartheta^{k} \\
\times\left(\Omega^{\natural}\right)^{\ell}\left(\alpha^{\natural}\right)^{r}\left(\beta^{\natural}\right)^{s}\left(\gamma^{\natural}\right)^{t} .
\end{gathered}
$$

Consider the above equation. We noted earlier that $S_{N}^{+}$is nonempty. By construction the scalar $\left\langle v, A^{i} B^{j} C^{k} \Omega^{\ell} \alpha^{r} \beta^{s} \gamma^{t}\right\rangle$ is nonzero for all $(i, j, k, \ell, r, s, t) \in S_{N}^{+}$. For $(i, j, k, \ell, r, s, t) \in S_{N}^{+}$, at least one of $i, j, k$ is zero since $i j k=0$. Moreover $i+k=N$ and $j+k \leq N$. For these constraints on $i, j, k$ the possible solutions for $(i, j, k)$ are

$$
(0,0, N),(1,0, N-1), \ldots,(N-1,0,1),(N, 0,0),(N, 1,0), \ldots,(N, N-1,0),(N, N, 0) .
$$

For the above values of $(i, j, k)$ the corresponding values of $\left(q^{-2 k} \vartheta+q^{2 k} \vartheta^{-1}\right)^{j} \vartheta^{k}$ are

$$
\vartheta^{N}, \vartheta^{N-1}, \ldots, \vartheta, 1, \vartheta+\vartheta^{-1}, \ldots,\left(\vartheta+\vartheta^{-1}\right)^{N-1},\left(\vartheta+\vartheta^{-1}\right)^{N} .
$$


The above line contains a sequence of Laurent polynomials in $\vartheta$. (9.6) below contains a sequence of Laurent polynomials in $\vartheta$. These two sequences are bases for the same vector space.

$$
\vartheta^{N}, \vartheta^{N-1}, \ldots, \vartheta, 1, \vartheta^{-1}, \ldots, \vartheta^{1-N}, \vartheta^{-N} .
$$

By these comments the equation (9.5) gives a nontrivial $\mathbb{F}$-linear dependency among

$$
\vartheta^{h}\left(\Omega^{\natural}\right)^{\ell}\left(\alpha^{\natural}\right)^{r}\left(\beta^{\natural}\right)^{s}\left(\gamma^{\natural}\right)^{t}, \quad \ell, r, s, t \in \mathbb{N}, \quad h \in \mathbb{Z}, \quad-N \leq h \leq N .
$$

In the above line we multiply each term by $\vartheta^{N}$ and obtain a nontrivial $\mathbb{F}$-linear dependency among

$$
\vartheta^{h}\left(\Omega^{\natural}\right)^{\ell}\left(\alpha^{\natural}\right)^{r}\left(\beta^{\natural}\right)^{s}\left(\gamma^{\natural}\right)^{t}, \quad h, \ell, r, s, t \in \mathbb{N}, \quad h \leq 2 N .
$$

This linear dependency contradicts Proposition 8.3, for the present case $M \leq N$.

Both cases yield a contradiction under the assumption that $J \neq 0$. Therefore $J=0$ so $\downarrow$ is injective.

\section{Comments on the $\mathrm{PSL}_{2}(\mathbb{Z})$ action}

Consider the injection $\downarrow: \Delta \rightarrow U \otimes \mathbb{F}\left[a^{ \pm 1}, b^{ \pm 1}, c^{ \pm 1}\right]$ from Theorem 2.16 and Theorem 2.18. Below Definition 2.2 we showed how $\mathrm{PSL}_{2}(\mathbb{Z})$ acts on $\Delta$ as a group of automorphisms. This $\mathrm{PSL}_{2}(\mathbb{Z})$ action induces a $\mathrm{PSL}_{2}(\mathbb{Z})$ action on the image $\Delta^{\natural}$. It is reasonable to ask whether this action extends to a $\operatorname{PSL}_{2}(\mathbb{Z})$ action on $U \otimes \mathbb{F}\left[a^{ \pm 1}, b^{ \pm 1}, c^{ \pm 1}\right]$ as a group of automorphisms. This extension does not quite work; let us examine what goes wrong.

Recall the generators $\rho, \sigma$ of $\mathrm{PSL}_{2}(\mathbb{Z})$ from below Definition 2.2. We will first consider the mathematics around $\sigma$.

One can readily check using Definition 2.6 that there exists an automorphism of $U$ that sends

$$
e \mapsto f, \quad f \mapsto e, \quad k^{ \pm 1} \mapsto k^{\mp 1}
$$

More generally, for any nonzero $\xi \in \mathbb{F}$ there exists an automorphism of $U$ that sends

$$
e \mapsto \xi f, \quad f \mapsto \xi^{-1} e, \quad k^{ \pm 1} \mapsto k^{\mp 1}
$$

The above automorphism swaps $U_{n}$ and $U_{-n}$ for all $n \in \mathbb{Z}$, where $\left\{U_{n}\right\}_{n \in \mathbb{Z}}$ is the $\mathbb{Z}$-grading of $U$ from below Lemma 6.1.

With these comments in mind we now consider the algebra $U \otimes \mathbb{F}\left[a^{ \pm 1}, b^{ \pm 1}, c^{ \pm 1}\right]$.

Lemma 10.1. There exists an automorphism $\tilde{\sigma}$ of $U \otimes \mathbb{F}\left[a^{ \pm 1}, b^{ \pm 1}, c^{ \pm 1}\right]$ that sends

$$
\begin{array}{llll}
e \otimes 1 \mapsto f \otimes a^{-1} b^{-1} c, & f \otimes 1 \mapsto e \otimes a b c^{-1}, & k \otimes 1 \mapsto k^{-1} \otimes 1, \\
1 \otimes a \mapsto 1 \otimes b, & 1 \otimes b \mapsto 1 \otimes a, & 1 \otimes c \mapsto 1 \otimes c .
\end{array}
$$

Moreover $\tilde{\sigma}^{2}=1$.

Proof. There exists an $\mathbb{F}$-algebra homomorphism $\tilde{\sigma}_{1}: U \rightarrow U \otimes \mathbb{F}\left[a^{ \pm 1}, b^{ \pm 1}, c^{ \pm 1}\right]$ that sends

$$
e \mapsto f \otimes a^{-1} b^{-1} c, \quad f \mapsto e \otimes a b c^{-1}, \quad k \mapsto k^{-1} \otimes 1 .
$$

There exists an $\mathbb{F}$-algebra homomorphism $\tilde{\sigma}_{2}: \mathbb{F}\left[a^{ \pm 1}, b^{ \pm 1}, c^{ \pm 1}\right] \rightarrow U \otimes \mathbb{F}\left[a^{ \pm 1}, b^{ \pm 1}, c^{ \pm 1}\right]$ that sends

$$
a \mapsto 1 \otimes b, \quad b \mapsto 1 \otimes a, \quad c \mapsto 1 \otimes c .
$$


Note that $\tilde{\sigma}_{1}(u) \tilde{\sigma}_{2}(f)=\tilde{\sigma}_{2}(f) \tilde{\sigma}_{1}(u)$ for all $u \in U$ and $f \in \mathbb{F}\left[a^{ \pm 1}, b^{ \pm 1}, c^{ \pm 1}\right]$. By these comments the map

$$
\begin{array}{ccc}
\tilde{\sigma}: \quad U \otimes \mathbb{F}\left[a^{ \pm 1}, b^{ \pm 1}, c^{ \pm 1}\right] & \rightarrow U \otimes \mathbb{F}\left[a^{ \pm 1}, b^{ \pm 1}, c^{ \pm 1}\right] \\
u \otimes f & \mapsto & \tilde{\sigma}_{1}(u) \tilde{\sigma}_{2}(f)
\end{array}
$$

is an $\mathbb{F}$-algebra homomorphism that satisfies $(10.1),(10.2)$. One checks $\tilde{\sigma}^{2}=1$. Therefore $\tilde{\sigma}$ is invertible and hence an automorphism.

Recall the $\mathbb{Z}$-grading of $U \otimes \mathbb{F}\left[a^{ \pm 1}, b^{ \pm 1}, c^{ \pm 1}\right]$ from above Lemma 7.1.

Lemma 10.2. The automorphism $\tilde{\sigma}$ of $U \otimes \mathbb{F}\left[a^{ \pm 1}, b^{ \pm 1}, c^{ \pm 1}\right]$ has the following effect on the $\mathbb{Z}$ grading. For $n \in \mathbb{Z}, \tilde{\sigma}$ swaps the homogeneous components with degree $n,-n$.

Proof. In (10.1), (10.2) we gave the action of $\tilde{\sigma}$ on some homogeneous generators for $U \otimes$ $\mathbb{F}\left[a^{ \pm 1}, b^{ \pm 1}, c^{ \pm 1}\right]$. For each generator its image under $\tilde{\sigma}$ is homogeneous. Moreover the generator and its image have opposite degree. The result follows.

We now consider the automorphism $\tilde{\sigma}$ of $U \otimes \mathbb{F}\left[a^{ \pm 1}, b^{ \pm 1}, c^{ \pm 1}\right]$ from the point of view of the equitable presentation.

Lemma 10.3. In the table below we display some elements $v$ of $U \otimes \mathbb{F}\left[a^{ \pm 1}, b^{ \pm 1}, c^{ \pm 1}\right]$. For each element $v$ we display the image $\tilde{\sigma}(v)$ under the map $\tilde{\sigma}$ from Lemma 10.1 .

\begin{tabular}{c|c}
$v$ & $\tilde{\sigma}(v)$ \\
\hline \hline$x \otimes 1$ & $y \otimes 1+\nu_{x} \otimes a^{-1} b^{-1} c$ \\
$y \otimes 1$ & $y^{-1} \otimes 1$ \\
$z \otimes 1$ & $y \otimes 1+\nu_{z} \otimes a b c^{-1}$ \\
\hline$\nu_{x} \otimes 1$ & $-q^{-1} \nu_{z} y^{-1} \otimes a b c^{-1}$ \\
$\nu_{y} \otimes 1$ & $-q \nu_{z} y \otimes a b c^{-1}+y \Lambda \otimes 1-\left(q+q^{-1}\right) y^{2} \otimes 1-q y \nu_{x} \otimes a^{-1} b^{-1} c$ \\
$\nu_{z} \otimes 1$ & $-q^{-1} y^{-1} \nu_{x} \otimes a^{-1} b^{-1} c$ \\
\hline$\Lambda \otimes 1$ & $\Lambda \otimes 1$
\end{tabular}

Proof. The images of $y \otimes 1, \nu_{x} \otimes 1, \nu_{z} \otimes 1$ are obtained from Lemma 10.1, using Note 3.2 and $y=k$. The images of $x \otimes 1$ and $z \otimes 1$ are now obtained using (6.2). The image of $\Lambda \otimes 1$ is found using Lemma 10.1 and (2.5). The image of $\nu_{y} \otimes 1$ is found using row $\nu_{y}$ in the table of Lemma 6.10.

Lemma 10.4. The map $\tilde{\sigma}$ from Lemma 10.1 sends

$$
\begin{aligned}
& \alpha^{\natural} \mapsto \beta^{\natural}, \quad \beta^{\natural} \mapsto \alpha^{\natural}, \quad \gamma^{\natural} \mapsto \gamma^{\natural}, \\
& A^{\natural} \mapsto B^{\natural}, \quad B^{\natural} \mapsto A^{\natural} .
\end{aligned}
$$

Proof. To verify (10.3), in the equations (4.4)-(4.6) apply $\tilde{\sigma}$ to each side, and evaluate the result using Lemma 10.1 and the fact that $\tilde{\sigma}$ fixes $\Lambda \otimes 1$. To verify (10.4) we refer to rows $A^{\natural}$ and $B^{\natural}$ of the table in Lemma 7.5. To each term in those rows, apply $\tilde{\sigma}$ and evaluate the result using Lemma 10.3.

Proposition 10.5. The following diagram commutes:

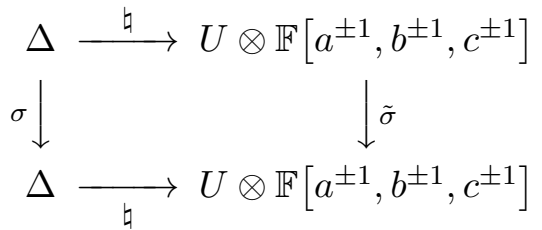


Proof. The $\mathbb{F}$-algebra $\Delta$ is generated by $A, B, C$. By this and (2.4) the $\mathbb{F}$-algebra $\Delta$ is generated by $A, B, \gamma$. Recall that $\sigma$ swap $A, B$ and fixes $\gamma$. By Lemma $10.4 \tilde{\sigma}$ swaps $A^{\natural}, B^{\natural}$ and fixes $\gamma^{\natural}$. The result follows.

Proposition 10.5 shows that the action of $\sigma$ on $\Delta^{\natural}$ extends to an automorphism $\tilde{\sigma}$ of $U \otimes$ $\mathbb{F}\left[a^{ \pm 1}, b^{ \pm 1}, c^{ \pm 1}\right]$ that has order 2 . So far so good. We now turn to the mathematics around $\rho$.

Consider the following variation on $U$.

Definition 10.6. Define the $\mathbb{F}$-algebra $U^{\prime}$ by generators $X, Y, Z$ and relations

$$
\frac{q X Y-q^{-1} Y X}{q-q^{-1}}=1, \quad \frac{q Y Z-q^{-1} Z Y}{q-q^{-1}}=1, \quad \frac{q Z X-q^{-1} X Z}{q-q^{-1}}=1 .
$$

The above presentation of $U^{\prime}$ resembles the equitable presentation of $U$, except that the generator $Y^{-1}$ is missing.

By construction there exists an $\mathbb{F}$-algebra homomorphism $\iota: U^{\prime} \rightarrow U$ that sends

$$
X \mapsto x, \quad Y \mapsto y, \quad Z \mapsto z
$$

We will need the fact that $\iota$ is injective. We will supply a proof shortly.

Lemma 10.7. The following is a basis for the $\mathbb{F}$-vector space $U$ :

$$
x^{h} y^{i} z^{j}, \quad h, j \in \mathbb{N}, \quad i \in \mathbb{Z} .
$$

Proof. For all $n \in \mathbb{N}$ let $V_{n}$ denote the subspace of $U$ spanned by those elements $e^{r} k^{s} f^{t}$ from (6.1) that satisfy $r+t=n$. By Lemma 6.1 the sum $U=\sum_{n=0}^{\infty} V_{n}$ is direct. For all $h, j \in \mathbb{N}$ and $i \in \mathbb{Z}$ let us write $x^{h} y^{i} z^{j}$ in terms of $e, k, f$. By Proposition 2.12 and Note 2.14,

$$
x=k^{-1}-e k^{-1} q^{-1}\left(q-q^{-1}\right), \quad y=k, \quad z=k^{-1}+f\left(q-q^{-1}\right) .
$$

Using this together with $k e=q^{2} e k$ and $k f=q^{-2} f k$, we find $x^{h} y^{i} z^{j} \in \sum_{n=0}^{h+j} V_{n}$ and

$$
x^{h} y^{i} z^{j}-(-1)^{h}\left(q-q^{-1}\right)^{h+j} q^{-h^{2}} e^{h} k^{i-h} f^{j} \in \sum_{n=0}^{h+j-1} V_{n} .
$$

The result follows since $\left\{e^{h} k^{i-h} f^{j} \mid h, j \in \mathbb{N}, i \in \mathbb{Z}\right\}$ is a basis for $U$.

Lemma 10.8. The following is a basis for the $\mathbb{F}$-vector space $U^{\prime}$ :

$$
X^{h} Y^{i} Z^{j}, \quad h, i, j \in \mathbb{N} .
$$

Proof. Using the relations in Definition 10.6 we routinely find that the elements (10.5) span $U^{\prime}$. The elements (10.5) are linearly independent, since their images under $\iota$ are linearly independent by Lemma 10.7. The result follows.

Lemma 10.9. The above map $\iota: U^{\prime} \rightarrow U$ is injective.

Proof. For the basis vectors (10.5) their images under $\iota$ are linearly independent by Lemma 10.7 . 
Consider the subalgebra of $U$ generated by $x, y, z$. This subalgebra is the image of $U^{\prime}$ under $\iota$. Invoking Lemma 10.9 we identify this subalgebra with $U^{\prime}$ via $\iota$. The elements $\nu_{x}, \nu_{y}, \nu_{z}$ are contained in $U^{\prime}$ by Definition 3.1, and $\Lambda \in U^{\prime}$ by Lemma 2.15. The algebra $U^{\prime} \otimes \mathbb{F}\left[a^{ \pm 1}, b^{ \pm 1}, c^{ \pm 1}\right]$ is the subalgebra of $U \otimes \mathbb{F}\left[a^{ \pm 1}, b^{ \pm 1}, c^{ \pm 1}\right]$ generated by

$$
x \otimes 1, \quad y \otimes 1, \quad z \otimes 1, \quad 1 \otimes a^{ \pm 1}, \quad 1 \otimes b^{ \pm 1}, \quad 1 \otimes c^{ \pm 1} .
$$

The next result clarifies how $U^{\prime} \otimes \mathbb{F}\left[a^{ \pm 1}, b^{ \pm 1}, c^{ \pm 1}\right]$ is related to $U \otimes \mathbb{F}\left[a^{ \pm 1}, b^{ \pm 1}, c^{ \pm 1}\right]$.

Lemma 10.10. The following is a basis for the $\mathbb{F}$-vector space $U \otimes \mathbb{F}\left[a^{ \pm 1}, b^{ \pm 1}, c^{ \pm 1}\right]$ :

$$
x^{h} y^{i} z^{j} \otimes a^{r} b^{s} c^{t}, \quad h, j \in \mathbb{N}, \quad i, r, s, t \in \mathbb{Z} .
$$

The following is a basis for the $\mathbb{F}$-vector space $U^{\prime} \otimes \mathbb{F}\left[a^{ \pm 1}, b^{ \pm 1}, c^{ \pm 1}\right]$ :

$$
x^{h} y^{i} z^{j} \otimes a^{r} b^{s} c^{t}, \quad h, i, j \in \mathbb{N}, \quad r, s, t \in \mathbb{Z} .
$$

Proof. Use Lemma 10.7 and Lemma 10.8.

By (4.1)-(4.3) the subalgebra $U^{\prime} \otimes \mathbb{F}\left[a^{ \pm 1}, b^{ \pm 1}, c^{ \pm 1}\right]$ contains $A^{\natural}, B^{\natural}, C^{\natural}$. Therefore $U^{\prime} \otimes$ $\mathbb{F}\left[a^{ \pm 1}, b^{ \pm 1}, c^{ \pm 1}\right]$ contains $\Delta^{\natural}$. Consequently we may view $\natural$ as an injection $\natural: \Delta \rightarrow U^{\prime} \otimes$ $\mathbb{F}\left[a^{ \pm 1}, b^{ \pm 1}, c^{ \pm 1}\right]$.

By Definition 10.6 there exists an automorphism of $U^{\prime}$ that sends $(x, y, z)$ to $(y, z, x)$. This automorphism fixes $\Lambda$ in view of Lemma 2.15. There is also an automorphism of $\mathbb{F}\left[a^{ \pm 1}, b^{ \pm 1}, c^{ \pm 1}\right]$ that sends $(a, b, c)$ to $(b, c, a)$. Combining these automorphisms we obtain the following.

Lemma 10.11. There exists an automorphism $\tilde{\rho}$ of $U^{\prime} \otimes \mathbb{F}\left[a^{ \pm 1}, b^{ \pm 1}, c^{ \pm 1}\right]$ that sends

$$
\begin{array}{lll}
x \otimes 1 \mapsto y \otimes 1, & y \otimes 1 \mapsto z \otimes 1, & z \otimes 1 \mapsto x \otimes 1, \\
1 \otimes a \mapsto 1 \otimes b, & 1 \otimes b \mapsto 1 \otimes c, & 1 \otimes c \mapsto 1 \otimes a .
\end{array}
$$

Moreover $\tilde{\rho}^{3}=1$.

Lemma 10.12. The automorphism $\tilde{\rho}$ from Lemma 10.11 sends

$$
A^{\natural} \mapsto B^{\natural}, \quad B^{\natural} \mapsto C^{\natural}, \quad C^{\natural} \mapsto A^{\natural} .
$$

Proof. Evaluate (4.1)-(4.3) using Lemma 10.11.

Lemma 10.13. The automorphism $\tilde{\rho}$ from Lemma 10.11 fixes $\Lambda \otimes 1$ and sends

$$
\alpha^{\natural} \mapsto \beta^{\natural}, \quad \beta^{\natural} \mapsto \gamma^{\natural}, \quad \gamma^{\natural} \mapsto \alpha^{\natural} .
$$

Proof. This is routinely checked using (4.4)-(4.6) and the comment about $\Lambda$ above Lemma 10.11.

Proposition 10.14. The following diagram commutes:

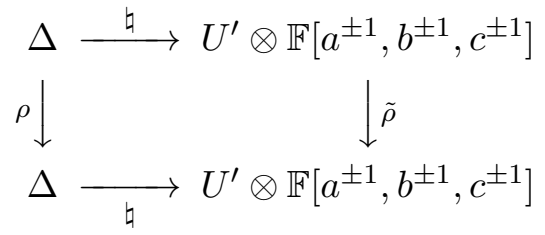

Proof. The algebra $\Delta$ is generated by $A, B, C$. Recall that $\rho$ cyclically permutes $A, B, C$. By Lemma $10.12 \tilde{\rho}$ of cyclically permutes $A^{\natural}, B^{\natural}, C^{\natural}$. The result follows. 
Proposition 10.14 shows that the action of $\rho$ on $\Delta^{\natural}$ extends to an automorphism $\tilde{\rho}$ of $U^{\prime} \otimes$ $\mathbb{F}\left[a^{ \pm 1}, b^{ \pm 1}, c^{ \pm 1}\right]$ that has order 3 . We now show that the automorphism $\tilde{\rho}$ of $U^{\prime} \otimes \mathbb{F}\left[a^{ \pm 1}, b^{ \pm 1}, c^{ \pm 1}\right]$ does not extend to an automorphism of $U \otimes \mathbb{F}\left[a^{ \pm 1}, b^{ \pm 1}, c^{ \pm 1}\right]$. For the moment assume that such an extension exists. Since it is an automorphism, it sends invertible elements to invertible elements. The element $y \otimes 1$ is invertible in $U \otimes \mathbb{F}\left[a^{ \pm 1}, b^{ \pm 1}, c^{ \pm 1}\right]$, with inverse $y^{-1} \otimes 1$. The element $z \otimes 1$ is not invertible in $U \otimes \mathbb{F}\left[a^{ \pm 1}, b^{ \pm 1}, c^{ \pm 1}\right]$, since $z$ is not invertible in $U$ by [9, Lemma 3.5]. This gives a contradiction since $\tilde{\rho}$ sends $y \otimes 1$ to $z \otimes 1$. Therefore the automorphism $\tilde{\rho}$ of $U^{\prime} \otimes \mathbb{F}\left[a^{ \pm 1}, b^{ \pm 1}, c^{ \pm 1}\right]$ does not extend to an automorphism of $U \otimes \mathbb{F}\left[a^{ \pm 1}, b^{ \pm 1}, c^{ \pm 1}\right]$.

In the above discussion we failed to obtain a $\mathrm{PSL}_{2}(\mathbb{Z})$ action on $U \otimes \mathbb{F}\left[a^{ \pm 1}, b^{ \pm 1}, c^{ \pm 1}\right]$. Perhaps we should search instead for a $\mathrm{PSL}_{2}(\mathbb{Z})$ action on $U^{\prime} \otimes \mathbb{F}\left[a^{ \pm 1}, b^{ \pm 1}, c^{ \pm 1}\right]$. Our extensions $\tilde{\sigma}$ and $\tilde{\rho}$ do not give such an action, for the following reason.

Lemma 10.15. The automorphism $\tilde{\sigma}$ of $U \otimes \mathbb{F}\left[a^{ \pm 1}, b^{ \pm 1}, c^{ \pm 1}\right]$ does not leave $U^{\prime} \otimes \mathbb{F}\left[a^{ \pm 1}, b^{ \pm 1}, c^{ \pm 1}\right]$ invariant.

Proof. By Lemma 10.3 the map $\tilde{\sigma}$ sends $y \otimes 1$ to $y^{-1} \otimes 1$. By Lemma 10.10 the subalgebra $U^{\prime} \otimes \mathbb{F}\left[a^{ \pm 1}, b^{ \pm 1}, c^{ \pm 1}\right]$ contains $y \otimes 1$ but not $y^{-1} \otimes 1$. The result follows.

Problem 10.16. Find an $\mathbb{F}$-algebra $\mathcal{A}$ with the following properties:

(i) There exists an injection of $\mathbb{F}$-algebras $\sharp: U \otimes \mathbb{F}\left[a^{ \pm 1}, b^{ \pm 1}, c^{ \pm 1}\right] \rightarrow \mathcal{A}$.

(ii) The algebra $\mathcal{A}$ has an automorphism $\hat{\sigma}$ of order 2 that makes the following diagram commute:

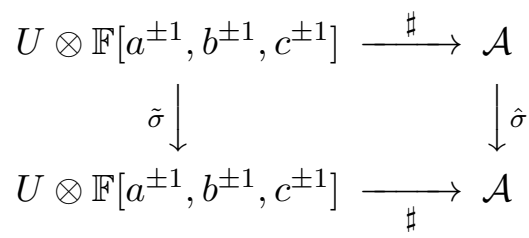

(iii) The algebra $\mathcal{A}$ has an automorphism $\hat{\rho}$ of order 3 that makes the following diagram commute:

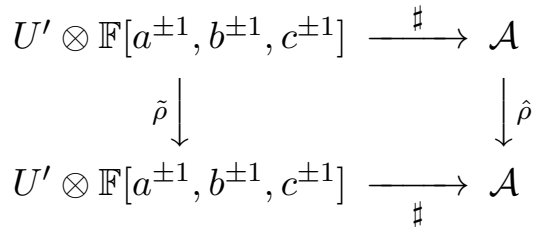

(iv) There does not exist a proper subalgebra of $\mathcal{A}$ that satisfies $(i)-(i i i)$ above.

The significance of the above problem is summarized below.

Proposition 10.17. Let $\mathcal{A}$ denote an $\mathbb{F}$-algebra that satisfies the four conditions of Problem 10.16. Then $\mathrm{PSL}_{2}(\mathbb{Z})$ acts on $\mathcal{A}$ as a group of automorphisms such that $\rho$ acts as $\hat{\rho}$ and $\sigma$ acts as $\hat{\sigma}$. Moreover the following diagram commutes for all $g \in \mathrm{PSL}_{2}(\mathbb{Z})$ :

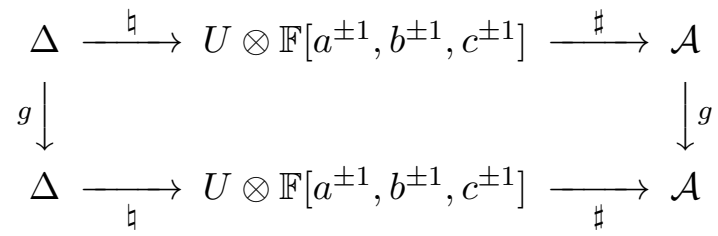

Proof. The $\mathrm{PSL}_{2}(\mathbb{Z})$ action on $\mathcal{A}$ exists by the construction. Concerning the diagram, without loss we may assume that $g=\sigma$ or $g=\rho$. For $g=\sigma$ the diagram commutes by Proposition 10.5 and Problem 10.16(ii). For $g=\rho$ the diagram commutes by Proposition 10.14 and Problem 10.16(iii). 


\section{References}

[1] Alnajjar H., Leonard pairs from the equitable generators of $U_{q}\left(\mathfrak{s l}_{2}\right)$, Dirasat Pure Sciences, Vol. 37, University of Jordan, 2010, available at http://www.ju.edu.jo/sites/Academic/h.najjar.

[2] Alnajjar H., Leonard pairs associated with the equitable generators of the quantum algebra $U_{q}\left(\mathfrak{s l}_{2}\right)$, Linear Multilinear Algebra 59 (2011), 1127-1142.

[3] Alperin R.C., Notes: $\mathrm{PSL}_{2}(\mathbb{Z})=\mathbb{Z}_{2} \star \mathbb{Z}_{3}$, Amer. Math. Monthly 100 (1993), 385-386.

[4] Askey R., Wilson J., Some basic hypergeometric polynomials that generalize Jacobi polynomials, Mem. Amer. Math. Soc. 54 (1985), no. 319.

[5] Baseilhac P., An integrable structure related with tridiagonal algebras, Nuclear Phys. B 705 (2005), 605-619, math-ph/0408025.

[6] Granovskiŭ Ya.I., Zhedanov A.S., Linear covariance algebra for $\mathrm{sl}_{q}(2)$, J. Phys. A: Math. Gen. 26 (1993), L357-L359.

[7] Ismail M., Classical and quantum orthogonal polynomials in one variable, Encyclopedia of Mathematics and its Applications, Vol. 98, Cambridge University Press, Cambridge, 2009.

[8] Ito T., Terwilliger P., Double affine Hecke algebras of rank 1 and the $\mathbb{Z}_{3}$-symmetric Askey-Wilson relations, SIGMA 6 (2010), 065, 9 pages, arXiv:1001.2764.

[9] Ito T., Terwilliger P., Weng C.-W., The quantum algebra $U_{q}\left(\mathfrak{s l}_{2}\right)$ and its equitable presentation, J. Algebra 298 (2006), 284-301, math.QA/0507477.

[10] Jantzen J.C., Lectures on quantum groups, Graduate Studies in Mathematics, Vol. 6, American Mathematical Society, Providence, RI, 1996.

[11] Koekoek R., Lesky P.A., Swarttouw R., Hypergeometric orthogonal polynomials and their q-analogues, Springer Monographs in Mathematics, Springer-Verlag, Berlin, 2010.

[12] Koornwinder T.H., The relationship between Zhedanov's algebra $A W(3)$ and the double affine Hecke algebra in the rank one case, SIGMA 3 (2007), 063, 15 pages, math.QA/0612730.

[13] Lavrenov A.N., Relativistic exactly solvable models, in Proceedings VIII International Conference on Symmetry Methods in Physics (Dubna, 1997), Phys. Atomic Nuclei 61 (1998), 1794-1796.

[14] Odake S., Sasaki R., Unified theory of exactly and quasiexactly solvable "discrete" quantum mechanics. I. Formalism, J. Math. Phys. 51 (2010), 083502, 24 pages, arXiv:0903.2604.

[15] Terwilliger P., Two linear transformations each tridiagonal with respect to an eigenbasis of the other, Linear Algebra Appl. 330 (2001), 149-203, math.RA/0406555.

[16] Terwilliger P., Two linear transformations each tridiagonal with respect to an eigenbasis of the other; comments on the parameter array, Des. Codes Cryptogr. 34 (2005), 307-332, math.RA/0306291.

[17] Terwilliger P., The universal Askey-Wilson algebra, SIGMA 7 (2011), 069, 24 pages, arXiv:1104.2813.

[18] Terwilliger P., Vidunas R., Leonard pairs and the Askey-Wilson relations, J. Algebra Appl. 3 (2004), 411426, math.QA/0305356.

[19] Wiegmann P.B., Zabrodin A.V., Algebraization of difference eigenvalue equations related to $U_{q}\left(s l_{2}\right), N u c l e a r$ Phys. B 451 (1995), 699-724, cond-mat/9501129.

[20] Zhedanov A.S., "Hidden symmetry" of the Askey-Wilson polynomials, Theoret. and Math. Phys. 89 (1991), $1146-1157$. 